\title{
Sirt1 activation protects the mouse renal medulla from oxidative injury
}

\author{
Wenjuan He,, ${ }^{1}$ Yingying Wang, ${ }^{1}$ Ming-Zhi Zhang, ${ }^{1}$ Li You, ${ }^{1,2}$ Linda S. Davis, ${ }^{1}$ Hong Fan, ${ }^{1,2}$ \\ Hai-Chun Yang,,2,3 Agnes B. Fogo,,1,3 Roy Zent,,1,4 Raymond C. Harris,1,4 \\ Matthew D. Breyer, ${ }^{5}$ and Chuan-Ming Hao, ${ }^{1,2}$
}

\begin{abstract}
${ }^{1}$ Nephrology Division, Vanderbilt University Medical Center School of Medicine, Nashville, Tennessee. ${ }^{2}$ Nephrology Division, Huashan Hospital, Fudan University, Shanghai, China. ${ }^{3}$ Department of Pathology, Vanderbilt University Medical Center, Nashville, Tennessee. ${ }^{4}$ Department of Medicine, Veterans Affairs Hospital, Nashville, Tennessee. ${ }^{5}$ Biotechnology Discovery Research, Lilly Research Laboratories, Eli Lilly and Company, Indianapolis, Indiana.
\end{abstract}

\begin{abstract}
Sirtuin 1 (Sirt1) is a NAD ${ }^{+}$-dependent deacetylase that exerts many of the pleiotropic effects of oxidative metabolism. Due to local hypoxia and hypertonicity, the renal medulla is subject to extreme oxidative stress. Here, we set out to investigate the role of Sirt1 in the kidney. Our initial analysis indicated that it was abundantly expressed in mouse renal medullary interstitial cells in vivo. Knocking down Sirt1 expression in primary mouse renal medullary interstitial cells substantially reduced cellular resistance to oxidative stress, while pharmacologic Sirt1 activation using either resveratrol or SRT2183 improved cell survival in response to oxidative stress. The unilateral ureteral obstruction (UUO) model of kidney injury induced markedly more renal apoptosis and fibrosis in Sirt1 ${ }^{+/-}$mice than in wild-type controls, while pharmacologic Sirt1 activation substantially attenuated apoptosis and fibrosis in wild-type mice. Moreover, Sirt1 deficiency attenuated oxidative stress-induced COX2 expression in cultured mouse renal medullary interstitial cells, and Sirt1 $1^{+/-}$mice displayed reduced UUO-induced COX2 expression in vivo. Conversely, Sirt1 activation increased renal medullary interstitial cell COX2 expression both in vitro and in vivo. Furthermore, exogenous $\mathrm{PGE}_{2}$ markedly reduced apoptosis in Sirt1-deficient renal medullary interstitial cells following oxidative stress. Taken together, these results identify Sirt1 as an important protective factor for mouse renal medullary interstitial cells following oxidative stress and suggest that the protective function of Sirt1 is partly attributable to its regulation of COX2 induction. We therefore suggest that Sirt1 provides a potential therapeutic target to minimize renal medullary cell damage following oxidative stress.
\end{abstract}

\section{Introduction}

The renal medulla, one of the harshest environments in the body, is characterized by excessive oxidative stress that results from rapidly changing interstitial tonicity as well as low blood flow and oxygen tension (1-3). Aging and diseased conditions such as the metabolic syndrome further increase oxidative stress in the kidney and are associated with reduced renal function $(4,5)$. Given that the renal medulla is critical for normal kidney function in regulating water and sodium balance as well as maintaining normal blood pressure (6-10), the development of strategies to maintain robust antioxidant mechanisms in the renal medulla is of paramount importance.

Mammalian sirtuin 1 (Sirt1) belongs to a highly conserved family of nicotinamide adenine dinucleotide-dependent $\left(\mathrm{NAD}^{+}\right.$-dependent) protein deacetylases and is widely expressed throughout almost all the mammalian organs $(11,12)$. Because of its dependency on cellular $\mathrm{NAD}^{+}$levels, Sirt 1 actively responds to redox reactions in cell metabolism (13). Intriguingly, activation of Sirt 1 by the naturally occurring compound resveratrol or newly developed specific activators increases lifespan of rodents, alleviates symptoms of metabolic syndrome, and protects mice from neurodegenerative diseases (14-17). The mechanisms underlying the beneficial effect of Sirt1 include an important antioxidant function (18-20).

In the present study, we characterized the histological distribution and the potential antioxidant role of Sirt1 in the kidney and

Conflict of interest: The authors have declared that no conflict of interest exists. Citation for this article: J Clin Invest. 2010;120(4):1056-1068. doi:10.1172/JCI41563. found that Sirt 1 is preferentially expressed in the inner medulla of the kidney, where it plays a critical role in promoting resistance of renal medullary interstitial cells to oxidative injury in vitro and in vivo. Sirt1-dependent cyclooxygenase-2 (COX2) induction may contribute to the protective function of Sirt1. Therefore, regulating Sirt 1 activity in vivo might have major implications in protecting the kidney from injury mediated by oxidative stress.

\section{Results}

Sirt1 is abundantly expressed in the renal medullary interstitial cells. Immunoblot analysis (Figure 1A) and quantitative real-time PCR (qRT-PCR) (Figure 1B, $P<0.05$ ) showed significantly higher levels of Sirt 1 expression in the renal medulla than in the renal cortex of wild-type C57BL/6 mice. Immunohistochemistry confirmed abundant nuclear Sirt1 immunoreactivity in the renal inner medulla (Figure 1C). Costaining for Sirt1 (red) and the renal segmental markers (green) showed abundant Sirt1-positive cells in the inner medullary interstitium (Figure 1D); Some aquaporin-2-positive (AQP2-positive) collecting duct cells also expressed Sirt1. We confirmed Sirt 1 expression in the renal medullary interstitial cells as Sirt1 (red) colocalized with a COX2 reporter EGFP that is specifically expressed in these cells (Figure 1E) (9).

Sirt 1 protects cultured renal medullary interstitial cells against oxidative stress. To define the level of oxidative stress within the medulla, we performed immunohistochemical studies for oxidative stress markers on mouse kidneys. Levels of nitrosylated tyrosine and 4-hydroxynonenal were greater in the renal medulla than in the renal cortex, with expression primarily in the renal medullary 
A

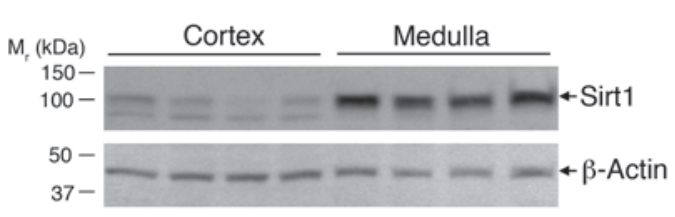

C
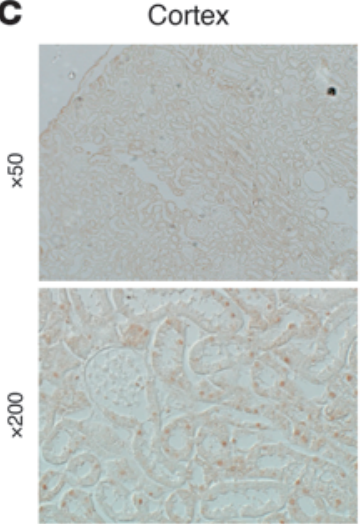

D

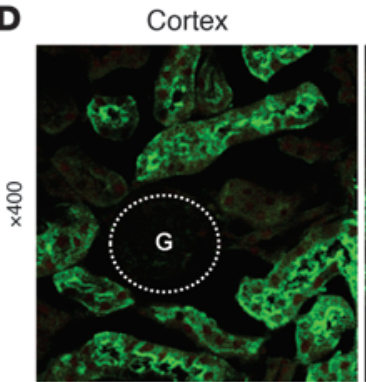

Sirt1/AQP1

E

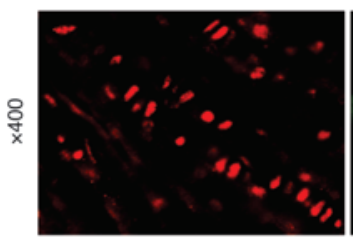

B

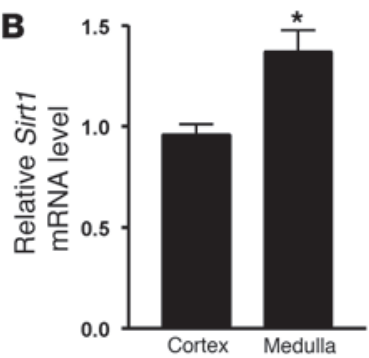

Outer medulla

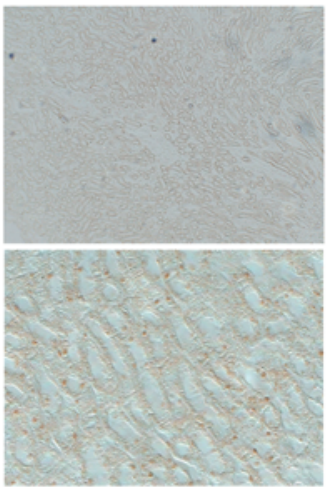

Outer medulla

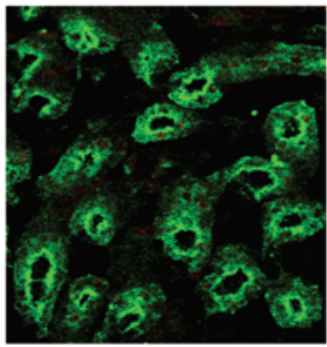

Sirt1/THP

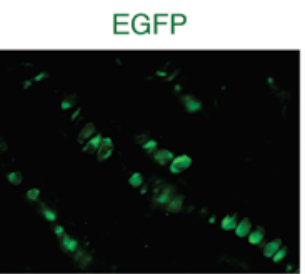

Inner medulla

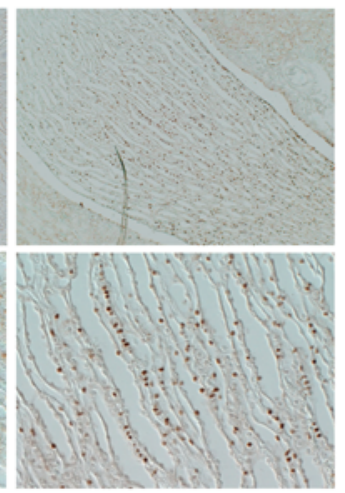

Inner medulla

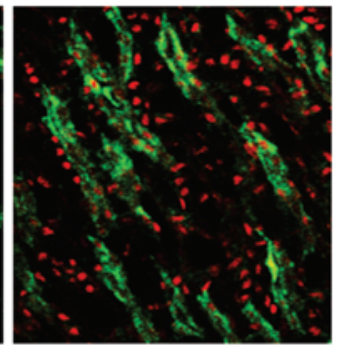

Sirt1/AQP2

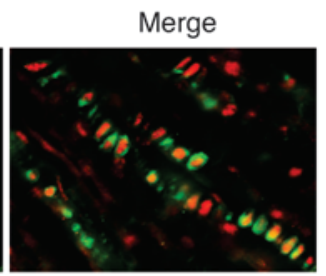

\section{Figure 1}

Sirt1 expression in mouse kidney. (A) Sirt1 protein expression in the C57BL/6J mouse renal medulla (including outer and inner medulla) and the renal cortical region was examined by immunoblot. Each lane represents a lysate from a single mouse. (B) Sirt1 mRNA expression in the renal medulla and the cortex was examined by qRT-PCR $(n=4$, $\left.{ }^{*} P<0.05\right)$. (C) Immunohistochemistry for Sirt1 expression in the mouse kidney. (D) Immunofluorescence costaining for Sirt1 (red) and renal segmental markers (green): AQP1 (proximal tubule), THP (thick ascending limb), AQP2 (collecting duct). $\mathrm{G}$, glomerulus. (E) Immunofluorescence stained Sirt1 (red) in the EGFP-positive RMICs of COX2 promoter-driven EGFP reporter transgenic mice. interstitium (Figure 2). Inner medullary interstitium appeared to have the highest levels of both oxidative stress markers (Figure 2).

Because Sirt 1 is abundantly expressed in renal medullary interstitial cells and there are high levels of oxidative stress markers in the renal medullary interstitium, we tested the role of Sirt1 in promoting cellular resistance to oxidative stress using primary cultured mouse renal medullary interstitial cells (RMICs). Downregulation of Sirt1 was achieved using a lentivirus carrying a Sirt1-selective shRNA. Sirt1 shRNA reduced endogenous Sirt1 protein expression by $85 \%$ (Figure $3 \mathrm{~A}, P<0.0001$ ). RT-PCR also showed that Sirt 1 shRNA decreased Sirt 1 mRNA expression, while Sirt 2 and Sirt3 mRNA expression remained unaltered (Figure 3B). Exposure of RMICs to oxidative stress ( $250 \mu \mathrm{M} \mathrm{H}_{2} \mathrm{O}_{2}, 12$ hours) significantly reduced cell viability, and this effect was accentuated by knockdown of Sirt 1 (Figure 3C, $22 \% \pm 4 \%$ versus $51 \% \pm 5 \%$,
$P<0.0001)$. TUNEL-positive apoptosis also increased after $\mathrm{H}_{2} \mathrm{O}_{2}$ treatment $(500 \mu \mathrm{M}, 6$ hours $)$ and was further increased by knockdown of Sirt1 (Figure 3E, $P<0.05$ ). $\mathrm{H}_{2} \mathrm{O}_{2}$ treatment-induced apoptosis that was further increased by knockdown of Sirt 1 was also confirmed by immunoblotting for cleaved caspase- 3 expression (Figure 3G, $P<0.001$ ).

We next examined whether the Sirt 1 activator resveratrol enhanced RMIC resistance to oxidative stress. Resveratrol $(5 \mu \mathrm{M})$ significantly enhanced the ability of RMICs to tolerate oxidative stress (500 $\mu \mathrm{M} \mathrm{H}_{2} \mathrm{O}_{2}, 12$ hours) (Figure 3D, 54\% $\pm 8 \%$ versus $35 \% \pm 3 \%, P<0.001)$. Knockdown of Sirt1 using shRNA abolished this protective effect (Figure 3D). Treatment of RMICs with SRT2183 $(5 \mu \mathrm{M})$, another specific and potent Sirt1 activator, also significantly reduced TUNEL-positive apoptosis after $\mathrm{H}_{2} \mathrm{O}_{2}$ treatment (Figure 3F, $P<0.001$ ). This was further confirmed by 


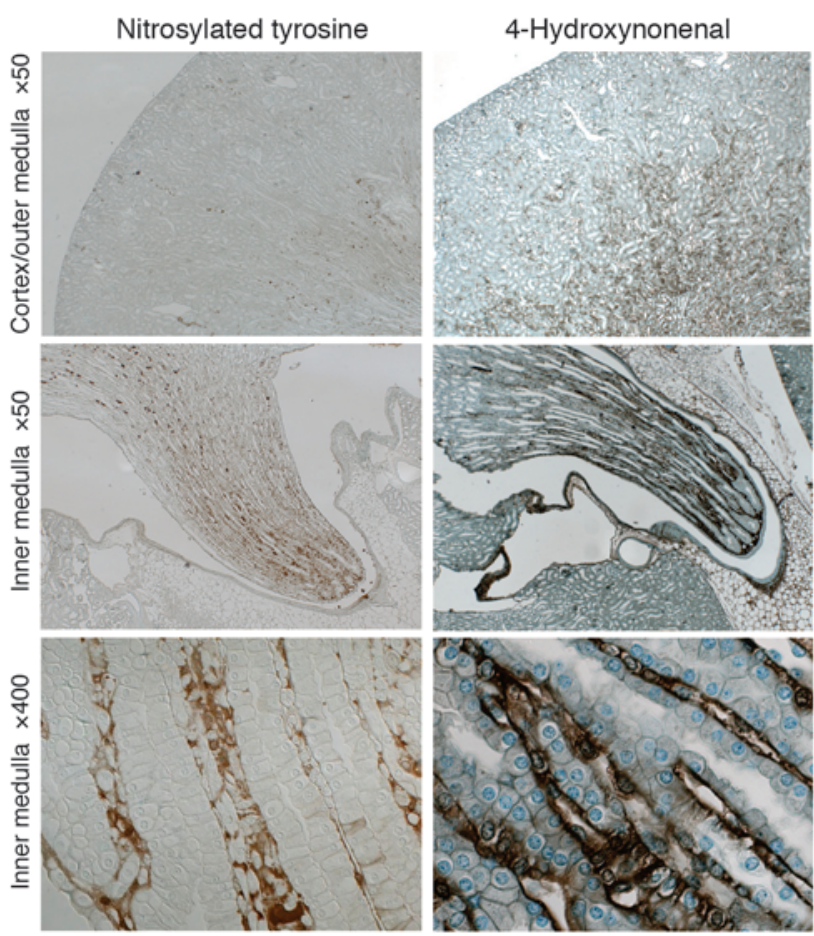

Figure 2

Markers of oxidative stress in mouse kidney. Immunohistochemistry using oxidative stress markers anti-nitrosylated tyrosine antibody (left panels) and anti-4-hydroxynonenal antibody (right panels). Panels at the bottom show high-magnification images of the renal inner medulla.

reduced $\mathrm{H}_{2} \mathrm{O}_{2}$-induced cleaved caspase- 3 level in RMICs treated with SRT2183 (Figure $3 \mathrm{H}, P<0.05$ ). These observations are consistent with an antioxidant role of Sirt1 in RMICs.

Sirt1 deficiency increases unilateral ureteral obstruction injury-induced apoptosis and fibrosis. To investigate the potential protective role of Sirt1 in kidney injury, we used the unilateral ureteral obstruction (UUO) model, which has been associated with increased oxidative stress $(21,22)$. Although homozygous Sirt1-knockout mice exhibit severe developmental defects (23), heterozygous Sirt1-knockout mice developed normally, and their kidneys were histologically normal (Supplemental Figure 1; supplemental material available online with this article; doi:10.1172/JCI41563DS1). qRT-PCR (Figure 4A, $P<0.01$ ) and immunoblot analysis (Figure 4B, $P<0.05$ ) confirmed significantly decreased Sirt 1 mRNA and protein expression in the kidney of heterozygous Sirt1-knockout (Sirt $1^{+-}$) mice. A significant increase in Sirt1 protein expression was observed on day 3 in the obstructed kidney of both $\operatorname{Sirt} 1^{+/+}$mice (Figure 4C, $P<0.0001)$ and Sirt $1^{+/-}$mice $(P<0.0001)$ (Figure 4C). In addition, Sirt1 was induced to a significantly lesser degree in the Sirt $1^{+/-}$than in the $\mathrm{Sirt}^{+/+}$mouse kidney (Figure 4C, $P<0.001$ ).

Renal cell apoptosis was seen predominantly in the renal medulla of mice after UUO (3 days) as assessed by TUNEL assay. TUNELpositive apoptosis was significantly greater in the renal medulla of Sirt $1^{+/-}$mice than that of Sirt $1^{+/+}$mice (Figure 5A, $P<0.05$ ). Increased apoptosis in the obstructed kidney of $\operatorname{Sirt} 1^{+/-}$mice was further confirmed by higher levels of cleaved caspase-3 expression when compared with Sirt ${ }^{+/+}$mice (Figure 5B, $P<0.01$ ). Seven days after UUO, Sirius red staining, a marker of collagen, was greater in the obstructed kidney of Sirt $1^{+/-}$versus $\operatorname{Sirt} 1^{+/+}$mice (Figure 5C, $P<0.05)$. This result was confirmed by higher levels of type I collagen (Col1) expression in the obstructed kidney of Sirt $1^{+/-}$mice (Figure 5D, $P<0.05$ ). Thus Sirt 1 deficiency increases renal apoptosis and fibrosis after UUO injury.

Sirt1 activation reduces UUO injury-induced apoptosis and fibrosis. In contrast to Sirt 1 deficiency, treatment of wild-type mice with the Sirt1 activator SRT1720 $(100 \mathrm{mg} / \mathrm{kg} / \mathrm{d})$ significantly reduced TUNEL-positive apoptosis in the medulla of the obstructed kidney after UUO (3 days) (Figure 6A, $P<0.05$ ). Reduced apoptosis was further confirmed by reduced cleaved caspase- 3 expression in the obstructed kidney of SRT1720-treated versus vehicle-treated mice (Figure 6B, $P<0.0001$ ). Furthermore, SRT1720 treatment significantly reduced Sirius red staining (Figure 6C, $P<0.05$ ) and Col1 expression in the obstructed kidney after UUO (7 days) (Figure $6 \mathrm{D}, P<0.05)$. Thus, increasing Sirt 1 activity decreases renal apoptosis and fibrosis after UUO injury, suggesting a protective role of Sirt 1 in the obstructed kidney.

Sirt1 regulates $R M I C$ COX 2 expression. Sirt 1 colocalizes with COX2 in RMICs, and COX2 activity is an important survival factor in RMICs (24-26). To further explore the mechanism by which Sirt1 activation protects RMICs from injury, we examined whether Sirt1 activity promoted expression of COX2 in RMICs. RMIC COX2 protein and mRNA expression was markedly increased by $\mathrm{H}_{2} \mathrm{O}_{2}$ (500 $\mu \mathrm{M}, 6$ hours) (Figure 7, A and B). $\mathrm{H}_{2} \mathrm{O}_{2}$-induced COX2 protein expression was nearly completely abolished by Sirt 1 shRNA (Figure 7A). Sirt1 shRNA also dramatically reduced $\mathrm{H}_{2} \mathrm{O}_{2}$-induced COX2 mRNA expression (Figure 7B, $P<0.0001$ ). Moreover, treatment of RMICs with the Sirt1 activator SRT2183 increased COX2 expression in a dose-dependent manner (Figure 7C). In contrast, neither $\mathrm{H}_{2} \mathrm{O}_{2}$ nor downregulation of Sirt 1 altered COX1 expression (Figure 7A), and Sirt1 activator did not induce COX1 expression (data not shown), supporting a specific effect of Sirt1 on COX2 expression. Furthermore, induction of COX2 by hypertonic stress was not affected by downregulation of Sirt1 (Supplemental Figure 2), suggesting that Sirt1 is specifically involved in the COX2 response after oxidative stress.

Since Sirt 1 activity regulates COX2 expression in vitro, we further investigated the role of Sirt 1 in regulating renal COX2 expression in vivo. Abundant COX2 induction (red) was observed primarily in the renal medulla of the obstructed kidney but not the contralateral kidney (Figure 8A). Costaining confirmed COX2 expression (red) in RMICs between AQP2-stained (green) collecting ducts (Figure 8A, right). Deletion of 1 allele of Sirt1 gene significantly reduced $\mathrm{COX} 2$ induction in the obstructed kidney after UUO (3 days) (Figure 8B, $P<0.05$ ). Furthermore, treatment of wild-type mice with the Sirt1 activator SRT1720 $(100 \mathrm{mg} / \mathrm{kg} / \mathrm{d})$ markedly induced COX2 expression (red) predominantly in the RMICs between AQP2-stained (green) collecting ducts (Figure $8 \mathrm{C})$. This result was further confirmed by increased renal COX2 expression in SRT1720-treated versus vehicle-treated mice as assessed by immunoblot (Figure 8D, $P<0.05$ ). Taken together, these in vivo observations are consistent with the in vitro results and support an important role for Sirt 1 in increasing COX2 expression in RMICs.

The mechanism linking Sirt1 and COX2 was further examined. Sirt 1 activity appears to regulate cultured RMIC COX2 expression at the transcriptional level, since Sirt 1 shRNA significantly blunted $\mathrm{H}_{2} \mathrm{O}_{2}$-induced ( $250 \mu \mathrm{M}, 12$ hours) COX2 luciferase reporter activation (Figure 7D, $P<0.0001$ ). To determine whether Sirt 1 physi- 

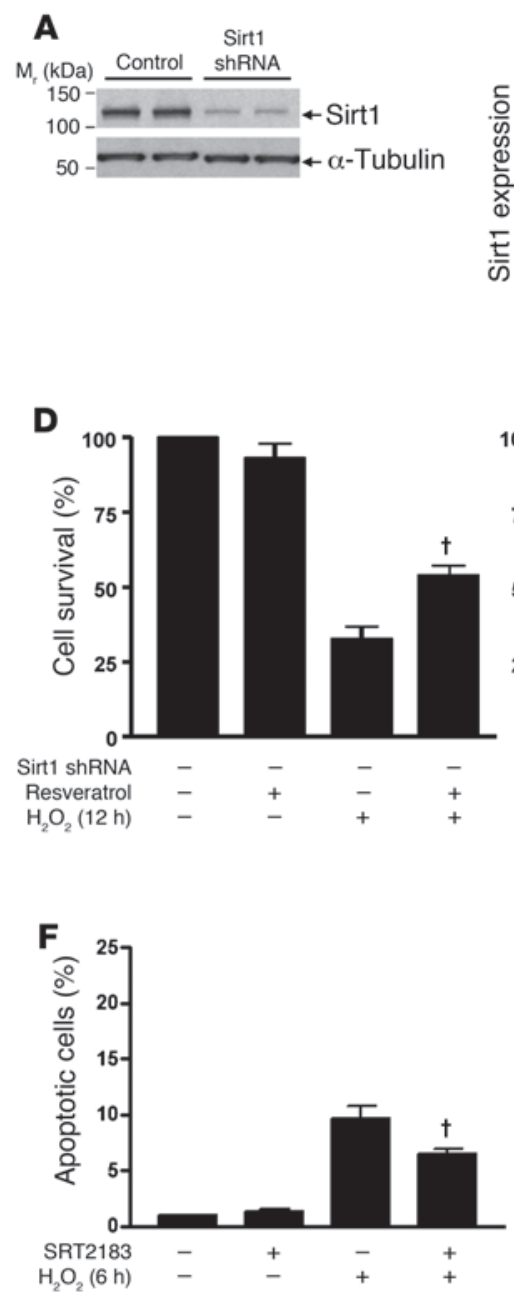

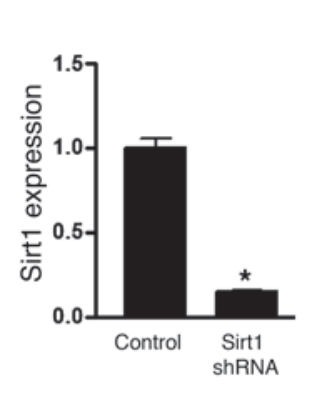

B

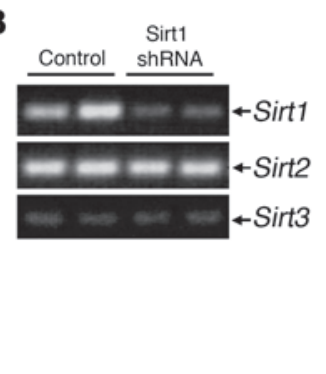

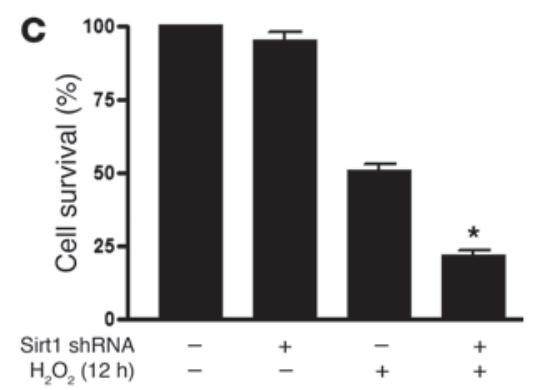

$\mathbf{E}$
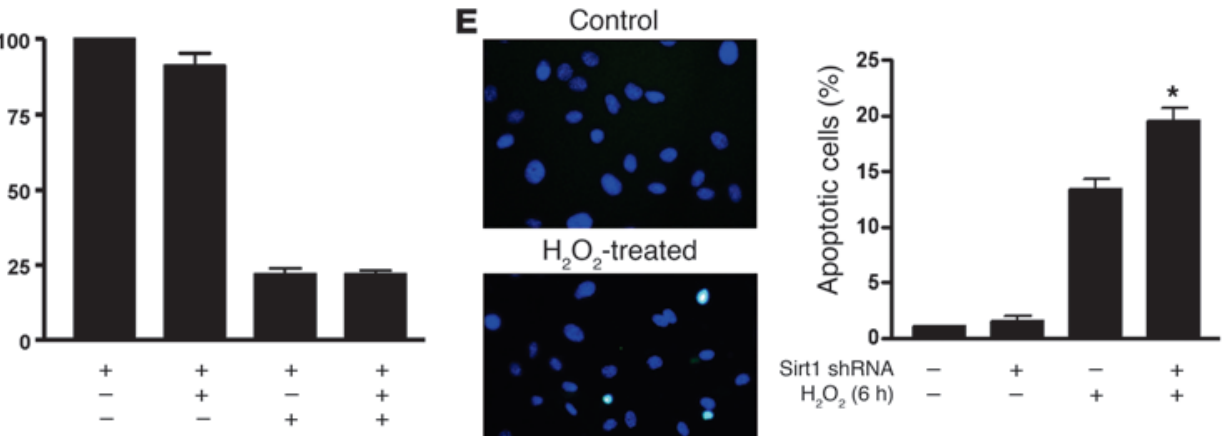

TUNEL/DAPI $\times 400$
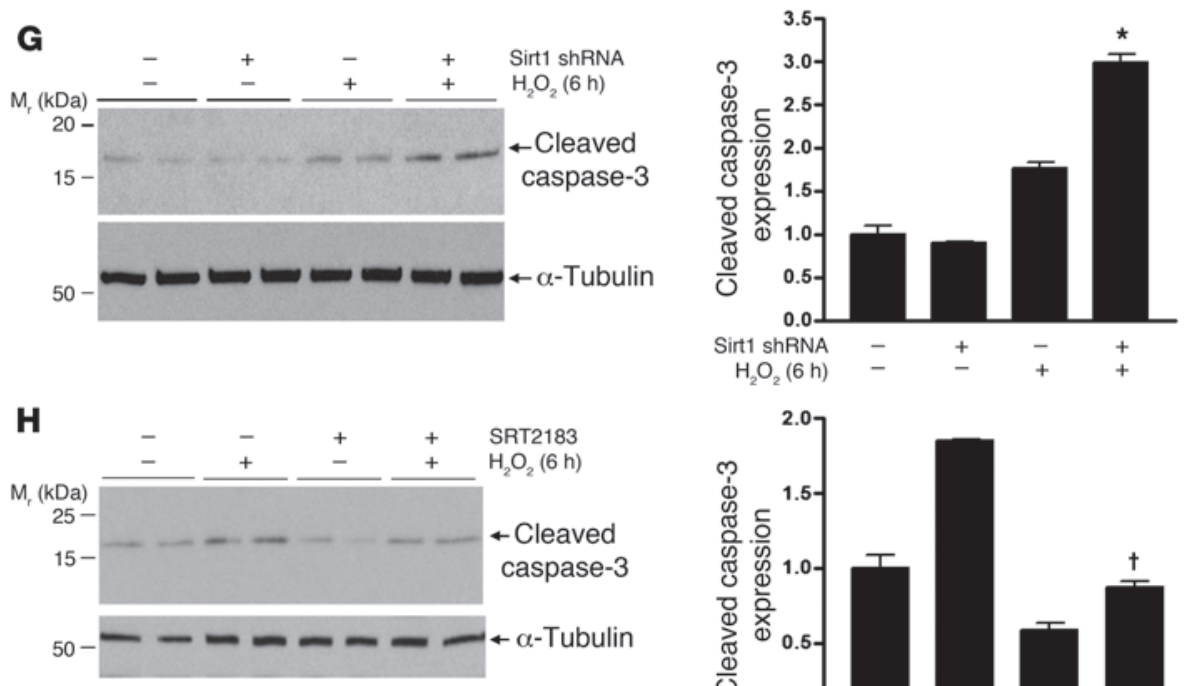

Figure 3

Sirt1 protects cultured RMICs from oxidative stress. (A and B) Primary mouse RMICs were infected with lentivirus carrying Sirt1-selective shRNA or control virus. Expression of Sirt1, Sirt2, and Sirt3 was examined by immunoblot (A, densitometry, $\left.n=4,{ }^{*} P<0.0001\right)$ and RT-PCR (B). (C and D) Control or Sirt1 shRNA-treated RMICs were pretreated with or without Sirt1 activator resveratrol $(5 \mu M)$ and challenged with $\mathrm{H}_{2} \mathrm{O}_{2}(250 \mu \mathrm{M})$ for 12 hours. Cell viability was examined by crystal violet staining $\left(n=6,{ }^{\star} P<0.0001\right.$ versus control virus-treated cells with $\mathrm{H}_{2} \mathrm{O}_{2}$; ${ }^{\dagger} P<0.001$ versus control virus-treated cells with $\mathrm{H}_{2} \mathrm{O}_{2}$ without resveratrol). (E and F) Control or Sirt1 shRNA-treated RMICs were pretreated with or without the Sirt1 activator SRT2183 $(5 \mu \mathrm{M})$ and challenged with $\mathrm{H}_{2} \mathrm{O}_{2}(500 \mu \mathrm{M})$ for 6 hours. Cell apoptosis was examined by TUNEL assay $(n=15$; ${ }^{*} P<0.05$ versus control virus-treated cells with $\mathrm{H}_{2} \mathrm{O}_{2}$; ${ }^{\dagger} P<0.001$ versus cells with $\mathrm{H}_{2} \mathrm{O}_{2}$ without SRT2183). (G and $\left.\mathbf{H}\right)$ Control or Sirt1 shRNAtreated RMICs were pretreated with or without the Sirt1 activator SRT2183 $(5 \mu \mathrm{M})$ and challenged with $\mathrm{H}_{2} \mathrm{O}_{2}(500 \mu \mathrm{M})$ for 6 hours. Expression of cleaved caspase-3, a marker of cellular apoptosis, was examined by immunoblot $\left(n=4\right.$, densitometry; ${ }^{*} P<0.001$ versus control virus-treated cells with $\mathrm{H}_{2} \mathrm{O}_{2} ;{ }^{\dagger} P<0.05$ versus cells with $\mathrm{H}_{2} \mathrm{O}_{2}$ without $\mathrm{SRT} 2183$ ).

cally associates with the COX2 promoter, we performed a ChIP assay on $\mathrm{H}_{2} \mathrm{O}_{2}$-treated RMICs. The presence of sequence from the mouse COX2 promoter region $\left(5^{\prime}\right.$ sequence -712 to -396$)$ in the DNAs immunoprecipitated with Sirt 1 antibody was confirmed by
PCR (Figure 7E). Sirt1-associated COX2 promoter sequence was detected in both nontreated cells and $\mathrm{H}_{2} \mathrm{O}_{2}$-treated cells, suggesting that Sirt 1 may be associated in a transcriptional complex on the COX2 promoter region. 


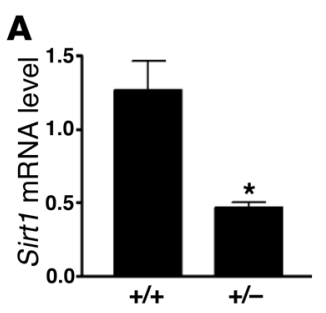

B

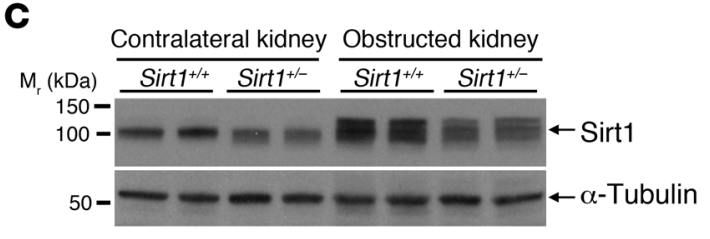

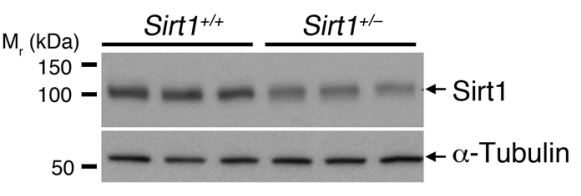

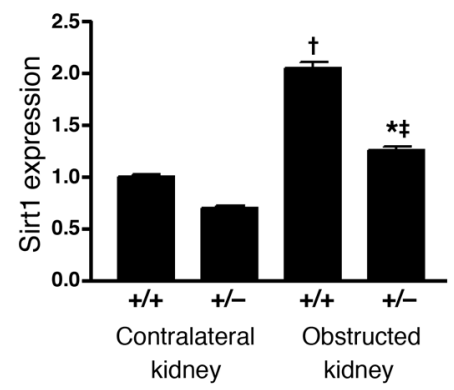

\section{Figure 4}

Reduced Sirt1 expression in the kidney of heterozygous Sirt1-knockout mice. (A) Relative Sirt1 mRNA expression level in the entire kidney of wild-type $\left(\right.$ Sirt1 $\left.{ }^{+/+}\right)$mice and heterozygous Sirt1knockout $\left(\right.$ Sirt1 $\left.{ }^{+-}\right)$mice was examined by qRT-PCR $\left(n=4,{ }^{*} P<0.01\right)$. (B) Sirt1 protein expression level in the entire kidney of Sirt1+/+ and Sirt1+/mice was examined by immunoblot $\left(n=6\right.$, densitometry, $\left.{ }^{*} P<0.05\right)$. (C) Sirt1 expression in the entire kidney of Sirt $1^{+/+}$and Sirt $1^{+/-}$mice 3 days after UUO was examined by immunoblot ( $n=4$, densitometry; ${ }^{\dagger} P<0.0001$ versus contralateral kidney of Sirt1+/+ mice; $\ddagger P<0.0001$ versus contralateral kidney of Sirt $1^{+/-}$mice; ${ }^{*} P<0.001$ versus obstructed kidney of Sirt1 ${ }^{+/+}$mice).
COX2-derived $P G E_{2}$ protects cultured RMICs from oxidative stress. The ability of RMICs to tolerate $\mathrm{H}_{2} \mathrm{O}_{2}$ is dependent on COX2 activity, since COX2 inhibition with SC58236 $(2.5 \mu \mathrm{M})$ reduced cell viability from $60 \% \pm 9 \%$ to $30 \% \pm 6 \%$ (Figure $9 \mathrm{~A}, P<0.0001$ ). Reduced cell resistance to oxidative stress by COX2 inhibition was further confirmed, as increased $\mathrm{H}_{2} \mathrm{O}_{2}$-induced TUNEL-positive apoptosis was observed in RMICs treated with SC58236 (2.5 $\mu \mathrm{M})$ (Figure 9C, $P<0.001)$. Conversely, preincubation of RMICs with $\mathrm{PGE}_{2}(100$ $\mathrm{nM}$ or $1 \mu \mathrm{M}$ ) dose-dependently restored viability of Sirt1-deficient RMICs exposed to $\mathrm{H}_{2} \mathrm{O}_{2}$ (Figure 9B, 69\% $\pm 6 \%, 82 \% \pm 9 \%$ versus $43 \% \pm 5 \%, P<0.05$ ). TUNEL assay (Figure 9D, $P<0.05$ ) and immunoblotting for cleaved caspase-3 expression (Figure 9E, $P<0.05$ ) further showed reduced $\mathrm{H}_{2} \mathrm{O}_{2}$-induced apoptosis in Sirt1-deficient RMICs treated with $\mathrm{PGE}_{2}(100 \mathrm{nM})$. Taken together, these observations support Sirt1-dependent COX2 induction as an important antioxidant mechanism in the RMICs.

\section{Discussion}

The present study demonstrates that the stress-responsive protein Sirt 1 is preferentially expressed in the renal inner medulla including medullary interstitial cells. Sirt 1 deficiency accentuates renal medullary cell apoptosis and renal fibrosis, and conversely, Sirt1 activation promotes the resistance of renal medullary cells to oxidative stress both in vitro and in vivo. One of the principal mechanisms by which Sirt 1 mediates its protective effects in RMICs is through increasing COX2 expression. These results therefore suggest that targeting Sirt 1 with pharmacological activators might improve renal function in conditions that induce oxidative stress in renal medulla including ureteral obstruction. It remains to be determined whether Sirt 1 activation also protects renal cell viability in other types of kidney diseases, including diabetic nephropathy and/or hypertensive nephropathy.

The antioxidant function of Sirt 1 has previously been reported in other organs such as the heart, neurons, and pancreatic $\beta$ cells (18-20). In the kidney, we found that Sirt 1 is abundantly expressed in the interstitial cells of renal inner medulla, where it plays a critical role against excessive oxidative stress. This result is consistent with the observation that there are high levels of oxidative stress markers under physiological conditions in the renal inner med- ullary interstitium. Multiple unique features of renal medullary interstitium are known to contribute to excessive oxidative stress, including low blood flow and oxygen tension as well as high osmotic stress due to high concentrations of sodium chloride and urea $(2,27)$. Besides oxidative stress, high concentrations of ammonia and $\mathrm{pH}$ changes in the renal medullary interstitium may also compromise cell viability (28). Whether the cytoprotective effect of Sirt 1 activity in the RMICs is limited to oxidative stress remains to be investigated. The present study also shows Sirt1 expression in some renal inner medullary collecting duct cells in vivo. Whether Sirt 1 exerts antioxidant function in these cells was not determined in the present study. Nonetheless, a physiological role for Sirt 1 in regulating the epithelial $\mathrm{Na}^{+}$channel $\alpha$-subunit $(\alpha-\mathrm{ENaC})$ expression has been reported in cultured renal inner medullary collecting duct cells (29). However, its relevance to the in vivo condition remains to be investigated. Furthermore, increased oxidative stress and phenotypic changes have been reported in aging kidneys $(4,30,31)$. Given the reported anti-stress and anti-aging nature of Sirt 1 function $(17,19,32,33)$, targeting of Sirt 1 by Sirt 1 activators may have therapeutic potential to protect the kidney from developing aging-related renal diseases.

The reported mechanisms by which Sirt 1 regulates cellular response to oxidative stress include modulation of the expression and/or activity of the cell cycle control protein FOXO; the DNA damage repair protein $\mathrm{Ku} 70$; and proteins involved in apoptosis, p53 and E2F1 (34). Here, we show that Sirt1-mediated COX2 induction and $\mathrm{PGE}_{2}$ production contribute to the protective function of Sirt 1 against oxidative stress in RMICs. Protective effects of COX2-derived $\mathrm{PGE}_{2}$ have been reported in several other studies in multiple organs and tissues such as the liver, kidney, retina, and neurons against injury of diverse causes (35-39). COX2 has been shown to protect the renal medulla from osmotic stress, and increased COX2 expression is a prerequisite for renal medullary cell survival from hypertonic stress $(1,24,25,40)$. However, a previous study failed to identify a beneficial effect of $\mathrm{PGE}_{2}$ on RMIC survival from hypertonic stress (40). Furthermore, by showing that Sirt1 regulates COX2 induction in the setting of oxidative stress and that $\mathrm{PGE}_{2}$ rescues oxidative stress-induced apoptosis in Sirt1deficient cells, our study places COX2 induction and $\mathrm{PGE}_{2}$ produc- 
A

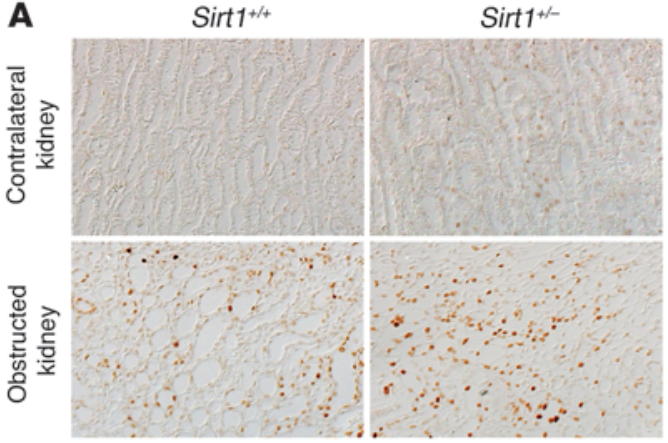

B

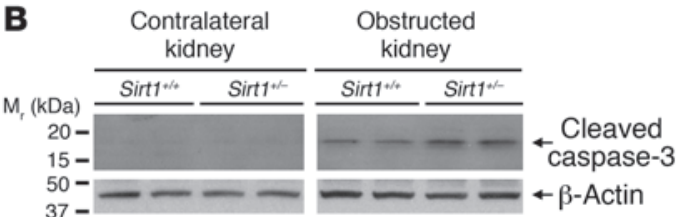

C

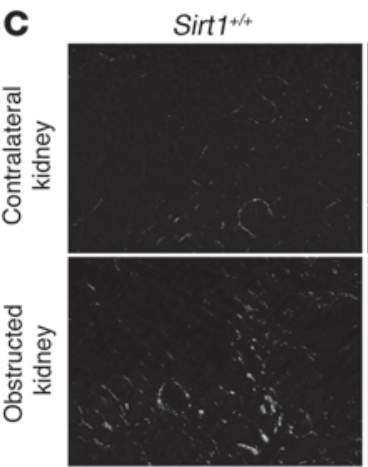

D

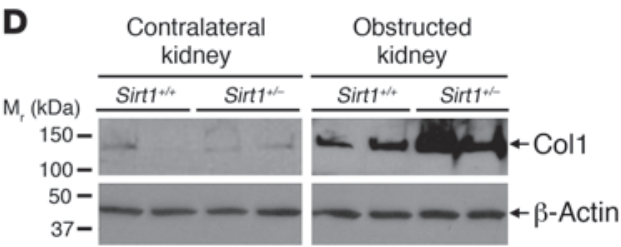

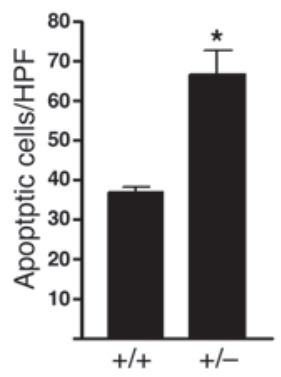
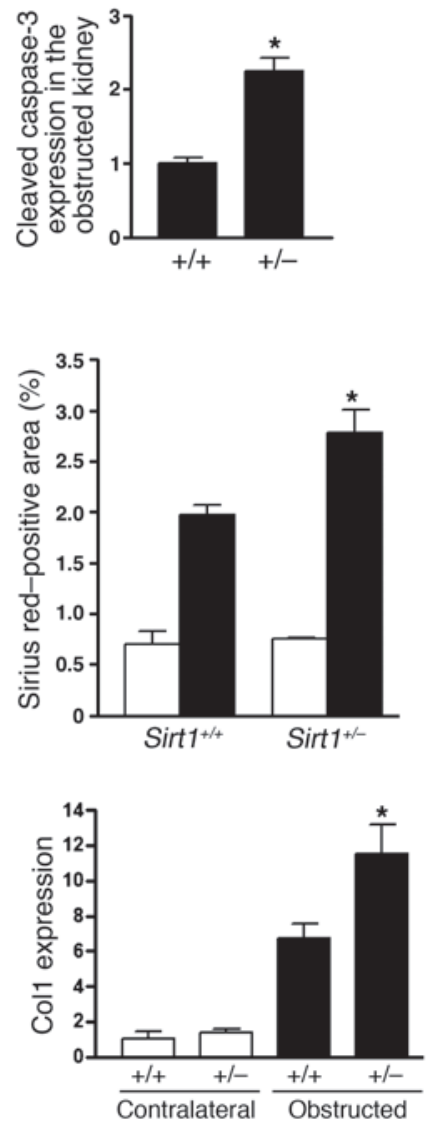

Figure 5

Sirt1 deficiency is associated with increased apoptosis and fibrosis in kidney subjected to ureteral obstruction. (A) Representative pictures (original magnification, $\times 200$ ) and quantification of TUNELpositive apoptosis in the renal medulla of Sirt $1^{+/+}$ mice and Sirt $1^{+/-}$mice 3 days after UUO $\left({ }^{\star} P<0.05\right)$. (B) Immunoblot for expression of the apoptosis marker cleaved caspase-3 in the entire kidney of Sirt $1^{+/+}$and Sirt1+/- mice 3 days after UUO $(n=4$, densitometry, ${ }^{*} P<0.01$ versus Sirt $1^{+/+}$mice). (C) Representative pictures (original magnification, $\times 200)$ and quantification of Sirius red staining on kidney sections of Sirt $1^{+/+}$and Sirt $1^{+/-}$mice 7 days after UUO ( ${ }^{*} P<0.05$ versus obstructed kidney of Sirt $1^{+/+}$mice). (D) Levels of Col1 protein expression in the entire kidney of Sirt1+/+ and Sirt1+/- mice 7 days after UUO were assessed by immunoblot ( $n=5$, densitometry, ${ }^{*} P<0.05$ versus obstructed kidney of Sirt1 ${ }^{+/+}$mice). tion downstream of Sirt 1 in the setting of oxidative stress in the RMICs. Because $\mathrm{PGE}_{2}$ is reported to exert its antiapoptotic effect through cAMP/PKA or PI3K/Akt signaling pathways (39, 41-43), these kinases may be responsible for the protective effect observed in our study. Given the fact that neither UUO injury nor the Sirt1 activator induces COX2 expression in renal medullary collecting duct cells in vivo, the Sirt1-dependent COX2 induction and $\mathrm{PGE}_{2}$ production may be a RMIC-specific protective mechanism. Since prostaglandins can act through both autocrine and paracrine mechanisms, it is possible that Sirt 1 in RMICs may protect adjacent renal tubules and vessels through locally diffused $\mathrm{PGE}_{2}$.

The present findings are supportive of a regulatory role of Sirt 1 on COX2 expression at the transcriptional level. Since Sirt 1 is a protein deacetylase, one possibility is that Sirt 1 deacetylates and activates DNA-binding proteins in a transcriptional complex on COX2 gene promoter, thus leading to activation of COX2 gene transcription. As Sirt1 knockdown suppressed the activation of the COX2 promoter-driven luciferase reporter in an exogenous plasmid (Figure 7D), Sirt1-regulated COX2 induction may be mediated through modification of transcription factors or cofactors. Several transcription factors and cofactors have been demonstrated to be deacetylase substrates of Sirt 1 in diverse tissues and cell types, suggesting a tissue and cell-specific manner of Sirt1 function. These transcription factors or cofactors include NF- $\kappa B$, p53, FOXO, and PGC-1 $\alpha$ (44-47). Notably, NF- $\mathrm{BB}$ is implicated in the regulation of COX2 gene transcription $(1,48)$. Unfortunately, our preliminary studies were unable to detect direct modification of NF-кB by Sirt 1 in the RMICs. Further experiments will be required to determine which molecule or molecules mediate the interaction between Sirt 1 and the COX2 promoter in the RMICs.

Sirt 1 responds to UUO injury as Sirt1 protein expression is induced in the kidney after ureteral obstruction (Figure 4C). Higher 
A

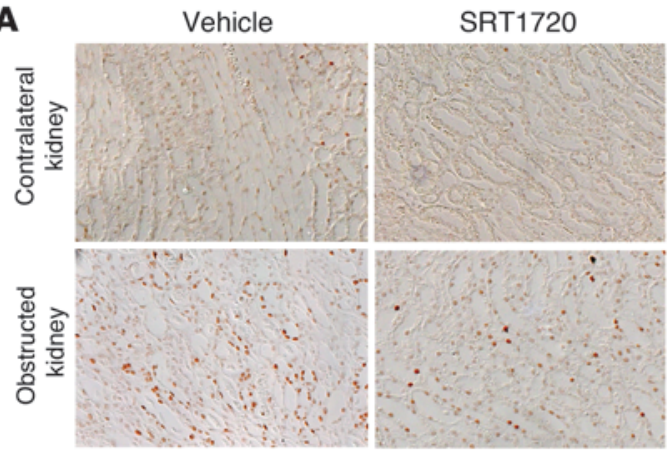

B

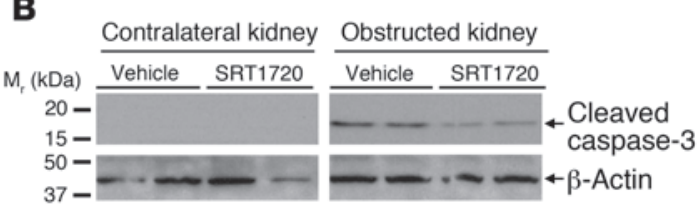

C
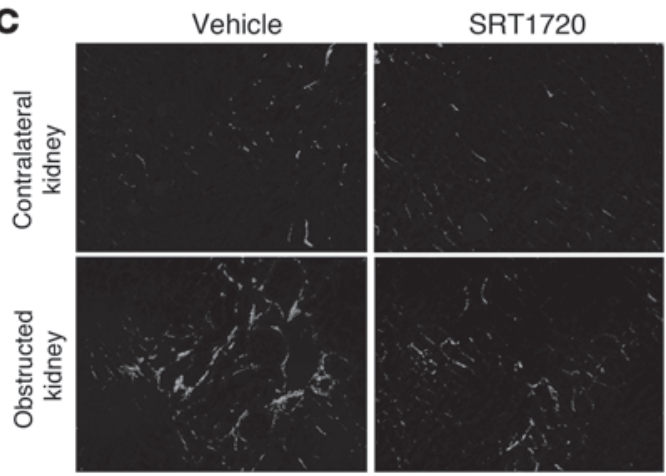

D

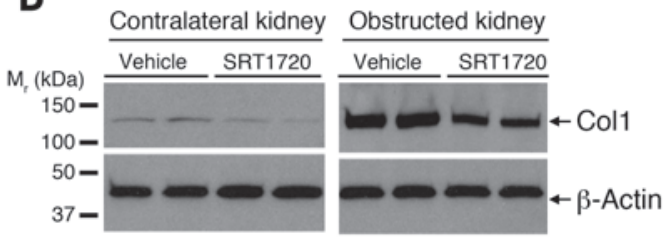

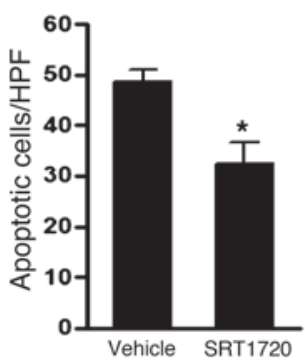
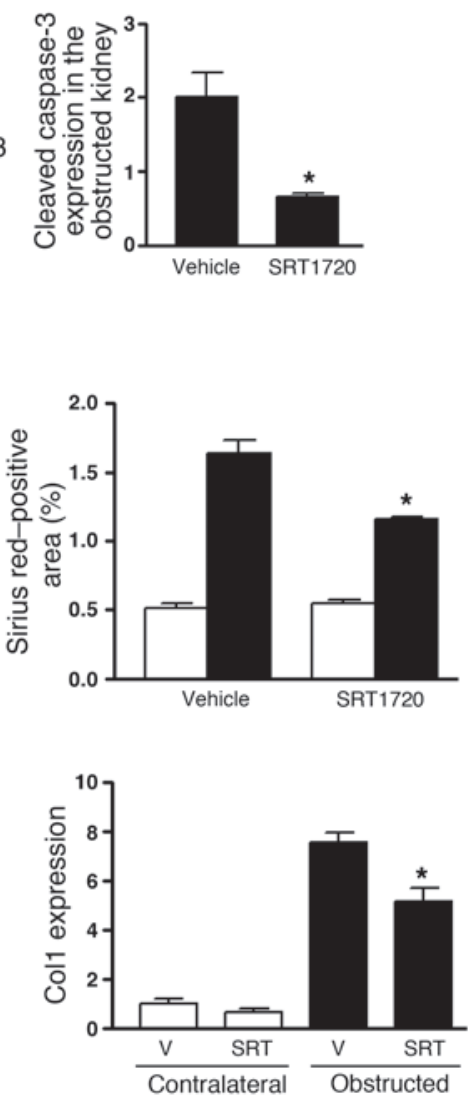

\section{Figure 6}

Sirt1 activation is associated with reduced apoptosis and fibrosis in kidney subjected to ureteral obstruction. (A) Representative images (original magnification, $\times 200$ ) and quantification of TUNEL-positive apoptosis in the renal medulla of vehicle-treated or Sirt1 activator SRT1720-treated (100 $\mathrm{mg} / \mathrm{kg} / \mathrm{d}$ ) wild-type mice 3 days after UUO $\left({ }^{*} P<0.05\right)$. (B) Immunoblot for cleaved caspase-3 expression in the entire kidney of vehicle-treated or SRT1720-treated wild-type mice 3 days after UUO $(n=4$, densitometry, ${ }^{*} P<0.0001$ versus obstructed kidney of vehicle-treated mice). (C) Representative images (original magnification, $\times 200$ ) and quantification of Sirius red staining on kidney sections of vehicle-treated or SRT1720treated mice 7 days after UUO $\left({ }^{\star} P<0.05\right.$ versus obstructed kidney of vehicle-treated mice). (D) Levels of Col1 protein expression in the entire kidney of vehicle-treated or SRT1720-treated wild-type mice 7 days after UUO were assessed by immunoblot $(n=5$, densitometry, ${ }^{*} P<0.05$ versus obstructed kidney of vehicle-treated mice). $\mathrm{V}$, vehicle; SRT, SRT1720. shifted bands recognized by anti-Sirt 1 antibody were present in the obstructed kidney, which is consistent with the possibility of protein modification of Sirt $1(49,50)$. Distribution of Sirt 1 in the obstructed kidneys remained similar to that in normal kidneys, with preferential expression in renal inner medulla (Supplemental Figure 3). Abundant Sirt1 expression was still present in the interstitial cells of the inner medulla. In addition, elevated Sirt1 immunoreactivity appeared in both renal cortical and medullary cells. Whether this reflects potential stress-responsive functions of Sirt 1 in other renal cell types besides RMICs remains uncertain.

The present findings show a beneficial role for Sirt 1 in protecting the kidney from fibrosis, which supports previous studies showing an antifibrotic effect of resveratrol (51-53). Although the detailed mechanisms of renal fibrosis after ureteral obstruction are still incompletely understood, fibrosis appears to be a maladaptive response to injury. If apoptosis and loss of resident renal medullary cells are considered as primary injury after UUO, reduced apoptosis and loss of resident renal medullary cells by Sirt 1 activity may consequently result in a reduced fibrotic response. In addition, the present observations that activation of Sirt 1 promotes RMIC viability and that Sirt1 activity reduces renal fibrosis after UUO injury further imply that RMICs actively protected by Sirt1 are probably not the cells contributing to fibrosis. Other cell populations such as cortical fibroblasts or circulating cells may migrate in and transform into fibroblasts.

In summary, the renal medullary environment is characterized by high oxidative stress. As the renal medulla is critical for the renal regulation of water and sodium balance as well as maintenance of normal blood pressure, maintaining robust antioxidant mechanisms in the renal medulla is of great importance. Our findings support an important role of Sirt1 activity, which is tightly modulated by oxidative metabolism, in protecting RMICs in the setting of oxidative stress. Recently, specific and potent small molecule Sirt 1 activators have shown therapeutic efficacy in alleviating 
A

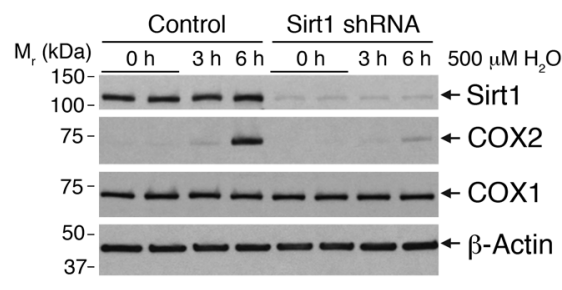

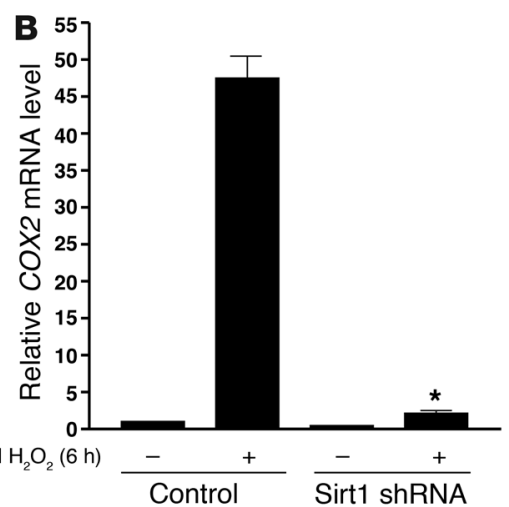

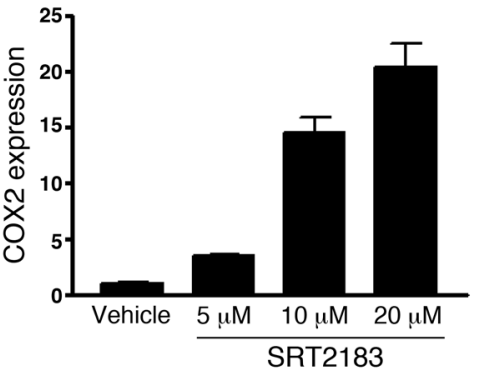

E

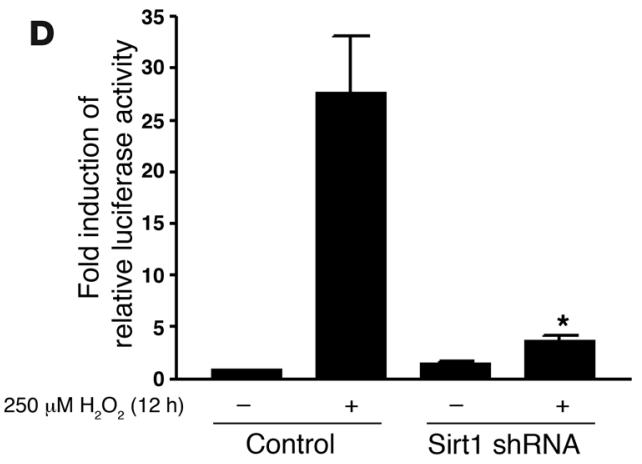

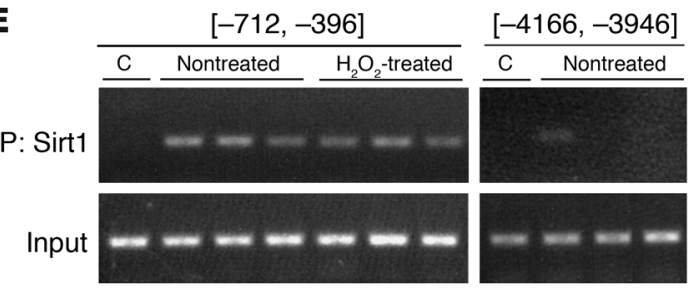

\section{Figure 7}

Sirt1 regulates COX2 expression in cultured RMICs. (A) Wild-type or Sirt1-knockdown cultured RMICs were challenged with $\mathrm{H}_{2} \mathrm{O}_{2}(500 \mu \mathrm{M})$ for 0 , 3, or 6 hours. Expression of Sirt1, COX2, and COX1 was examined by immunoblot. (B) Wild-type or Sirt1-knockdown RMICs were challenged with $\mathrm{H}_{2} \mathrm{O}_{2}(500 \mu \mathrm{M})$ for 0 or 6 hours. COX2 mRNA expression was assessed by qRT-PCR $\left(n=4,{ }^{\star} P<0.0001\right.$ versus wild-type cells with $\left.\mathrm{H}_{2} \mathrm{O}_{2}\right)$. (C) RMICs were treated with the Sirt1 activator SRT2183 $(0,5,10,20 \mu \mathrm{M})$ for 8 hours, and COX2 expression was examined by immunoblot $(n=4)$. (D) COX2 luciferase reporter activity was measured in wild-type or Sirt1-knockdown RMICs with $\mathrm{H}_{2} \mathrm{O}_{2}(250 \mu \mathrm{M})$ for 0 or 12 hours by using the Dual Luciferase assay kit $\left(n=12,{ }^{*} P<0.0001\right.$ versus wild-type cells with $\left.\mathrm{H}_{2} \mathrm{O}_{2}\right)$. (E) ChIP assay was performed on nontreated or $\mathrm{H}_{2} \mathrm{O}_{2}$-treated $(500 \mu M$, 3 hours) RMICs using anti-Sirt1 antibody and PCR primers recognizing mouse COX2 5' sequences upstream of the transcription start site [-712, $-396]$ or $[-4,166,-3,946]$. C, control: immunoprecipitation with normal rabbit IgG. Data are representative of 3 independent experiments.

symptoms in metabolic syndrome and neurodegenerative diseases. Our study suggests that targeting Sirt1 with Sirt1 activators may also be a potential therapeutic strategy for minimizing or preventing renal damage resulting from increased oxidative stress. Such conditions include metabolic syndrome and aging.

\section{Methods}

Animals. Mice were maintained in the animal facility of Vanderbilt University Medical Center, where they were housed in a constant-temperature room with a 12-hour dark/12-hour light circle and allowed free access to standard rodent chow and water. Wild-type C57BL/6J male mice at 8 weeks of age were obtained from The Jackson Laboratory. The floxed Sirt 1 mouse was generated by Yansong Gu et al. (Harvard University Medi- cal School, Boston, Massachusetts) (23) and deposited at The Jackson Laboratory (strain name: B6;129-Sirt ${ }^{\text {tm } 1 Y g u} / \mathrm{J}$ ). Mice with 1 allele of Sirt1 gene deletion were obtained by crossing a Sirt $11^{f /+}$ mouse with a universal Cre mouse (EIIA-Cre mice on a C57BL/6J background; provided by Richard M. Breyer, Vanderbilt University). This mouse was further bred with C57BL/6J to generate heterozygous Sirt1-knockout mice (Sirt1 $\left.{ }^{+-}\right)$and their wild-type littermates $\left(\operatorname{Sirt1}^{+/+}\right)$. Male mice between 8 and 10 weeks of age were used in the present study. All animal studies were approved by the Institutional Animal Care and Use Committees of Vanderbilt University. Kidney specimens were collected for immunoblot, qPT-PCR, and immunohistochemistry ( $n=3$ in each group).

Compounds. Sirt 1 activators SRT1720 and SRT2183 were provided by Christoph Westphal and Jill Milne (Sirtris, a GSK company, Cambridge, 
A Contralateral kidney

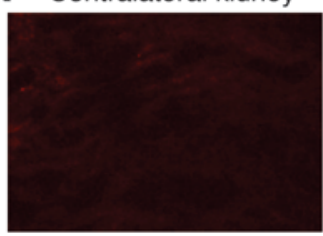

$\operatorname{cox} 2 \times 200$

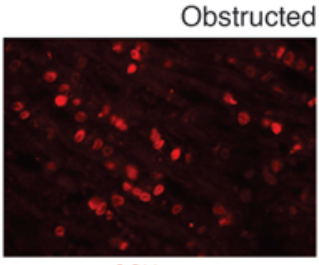

$\operatorname{cox} 2 \times 200$

B

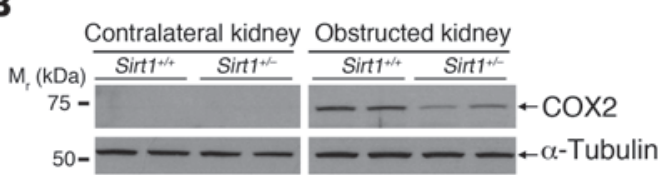

C

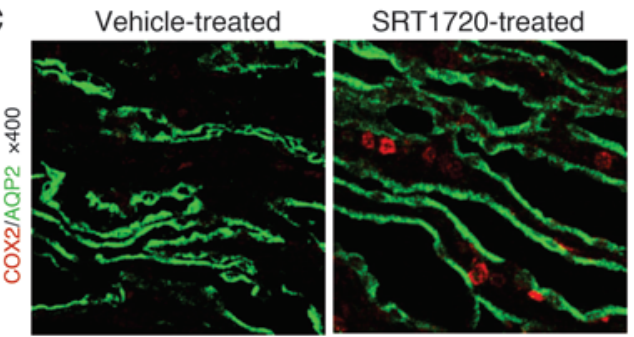

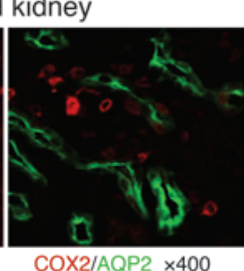

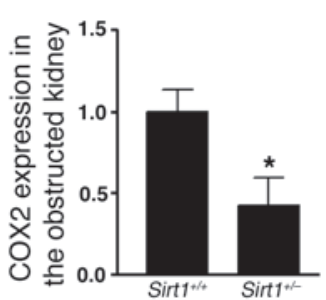

\section{Figure 8}

Sirt1 regulates COX2 expression in RMICs in vivo. (A) Representative immunofluorescence images show COX2 induction (red) in the renal medulla of the obstructed kidney of wild-type mice 3 days after UUO. Costaining further shows induced COX2 (red) in RMICs between AQP2positive (green) collecting ducts (right panel). (B) Immunoblot for COX2 expression in the entire kidney of Sirt1+/+ and Sirt $1^{+-}$mice 3 days after UUO $(n=5$, densitometry, ${ }^{*} P<0.05$ versus obstructed kidney of Sirt $1^{+/+}$mice). (C) Wild-type mice were treated with vehicle or Sirt1 activator SRT1720 (100 mg/kg/d) for 5 days. Immunofluorescence costaining shows that SRT1720 induces COX2 expression (red) in RMICs between AQP2-positive (green) collecting ducts. (D) COX2 expression in the entire kidney of vehicle or SRT1720-treated mice was examined by immunoblot $\left(n=4\right.$, densitometry, ${ }^{\star} P<0.05$ versus vehicle-treated mice).
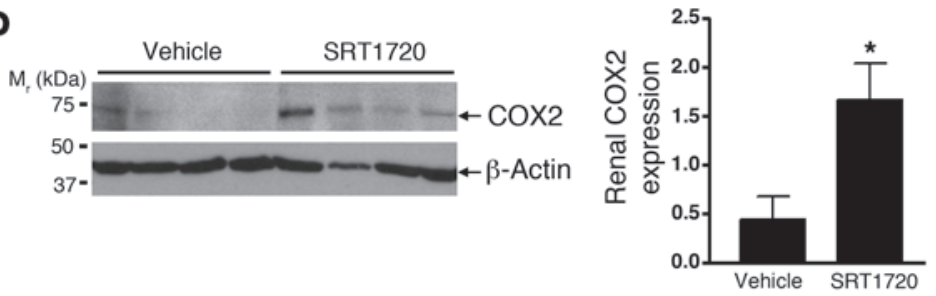

Massachusetts). For $1 \mathrm{ml}$ of $50 \mathrm{mg} / \mathrm{ml}$ stock dosing solution used for in vivo studies, $50 \mathrm{mg}$ of SRT1720 was added into $400 \mu \mathrm{l}$ PEG400 and vortexed to form a homogenous suspension. Tween-80 (5 $\mu \mathrm{l})$ was added into $595 \mu \mathrm{l}$ water and vortexed. Then Tween-80/water was added into SRT1720/PEG400 and vortexed until homogeneous. In about 5-10 minutes, the suspension became translucent-opaque. The final vehicle composition was $40 \%$ PEG $400,0.5 \%$ Tween-80, and 59.5\% water. SRT1720 (100 $\mathrm{mg} / \mathrm{kg}$ body weight) was given to mice by oral gavage once a day according to the company's recommendation. For in vitro studies, SRT compounds were dissolved in DMSO. A final concentration of 0.5-10 $\mu$ M SRT1720 and a final concentration of 1-20 $\mu \mathrm{M}$ SRT2 183 were used.

COX2 selective inhibitor SC58236 was provided by Karen Siebert and Peter Isakson (Pfizer/Searle, New York, New York). For in vitro studies, compounds were dissolved in DMSO. A final concentration of $0.5 \mu \mathrm{M}$ or $2.5 \mu \mathrm{M}$ was used.

UUO. Mice (male, 8 weeks old) were anesthetized with $50 \mathrm{mg} / \mathrm{kg}$ body weight ketamine and $100 \mathrm{mg} / \mathrm{kg}$ body weight xylazine. The left ureter was exposed via a lateral incision and ligated by 2 sutures at the level of the lower renal pole. Mice were sacrificed at day 3 or 7 after the operation. Both contralateral and obstructed kidneys were collected and assessed for gene expression, apoptosis, and fibrosis.

Immunoblot. Protein concentration was determined using the bicinchoninic acid protein assay (Sigma-Aldrich). Fifty micrograms of proteins were loaded in each lane of a $10 \%$ SDS-PAGE minigel and run at $100 \mathrm{~V}$. Proteins were transferred to a PVDF membrane at $100 \mathrm{~V}$ for 1 hour on ice.
The membrane was washed 3 times with TBST (50 mM Tris, $\mathrm{pH} 7.5,150$ $\mathrm{mM} \mathrm{NaCl}, 0.05 \%$ Tween-20), incubated in blocking buffer $(150 \mathrm{mM} \mathrm{NaCl}$, $50 \mathrm{mM}$ Tris, $0.05 \%$ Tween-20, and 5\% Carnation nonfat dry milk, $\mathrm{pH} 7.5$ ) for 1 hour at room temperature, and then incubated with primary antibody in blocking buffer overnight at $4^{\circ} \mathrm{C}$. The primary antibodies used were: anti-Sirt1 antibody (Millipore rabbit polyclonal, 1:1,000; SigmaAldrich mouse monoclonal, 1:2,000), anti-cleaved caspase-3 antibody (Cell Signaling rabbit monoclonal, 1:200), anti-COX2 antibody (Cayman rabbit polyclonal, 1:1,000), anti-COX1 antibody (Cayman rabbit polyclonal, 1:1,000), anti-Col1 antibody (MD Biosciences rabbit polyclonal, 1:10,000), anti- $\beta$-actin antibody (Jackson ImmunoResearch Laboratories mouse monoclonal, 1:5,000), and anti- $\alpha$-tubulin antibody (Sigma-Aldrich mouse monoclonal, 1:2,000). After 3 washes, the membrane was incubated with horseradish peroxidase-conjugated secondary antibody (Jackson ImmunoResearch Laboratories, 1:5,000) for 1 hour at room temperature, followed by 3 washes with TBST. Antibody labeling was visualized by the addition of chemiluminescence reagent (PerkinElmer Life Sciences), and the membrane was exposed to Kodak XAR-5 film. Due to heterogeneous expression of Sirt 1 in the kidney as well as distorted morphology of the obstructed kidney, tissue sampling (dissecting cortex from medulla) could easily have introduced artifacts into the data. For this reason, the entire kidney was homogenized, and whole kidney lysates were used for all the immunoblot studies in Figures 4-6 and 8.

Immunohistochemistry. Deparaffinized 5 - $\mu \mathrm{m}$ sections were briefly incubated with $3 \% \mathrm{H}_{2} \mathrm{O}_{2}$ and then with primary antibody for 60 minutes, rinsed with 

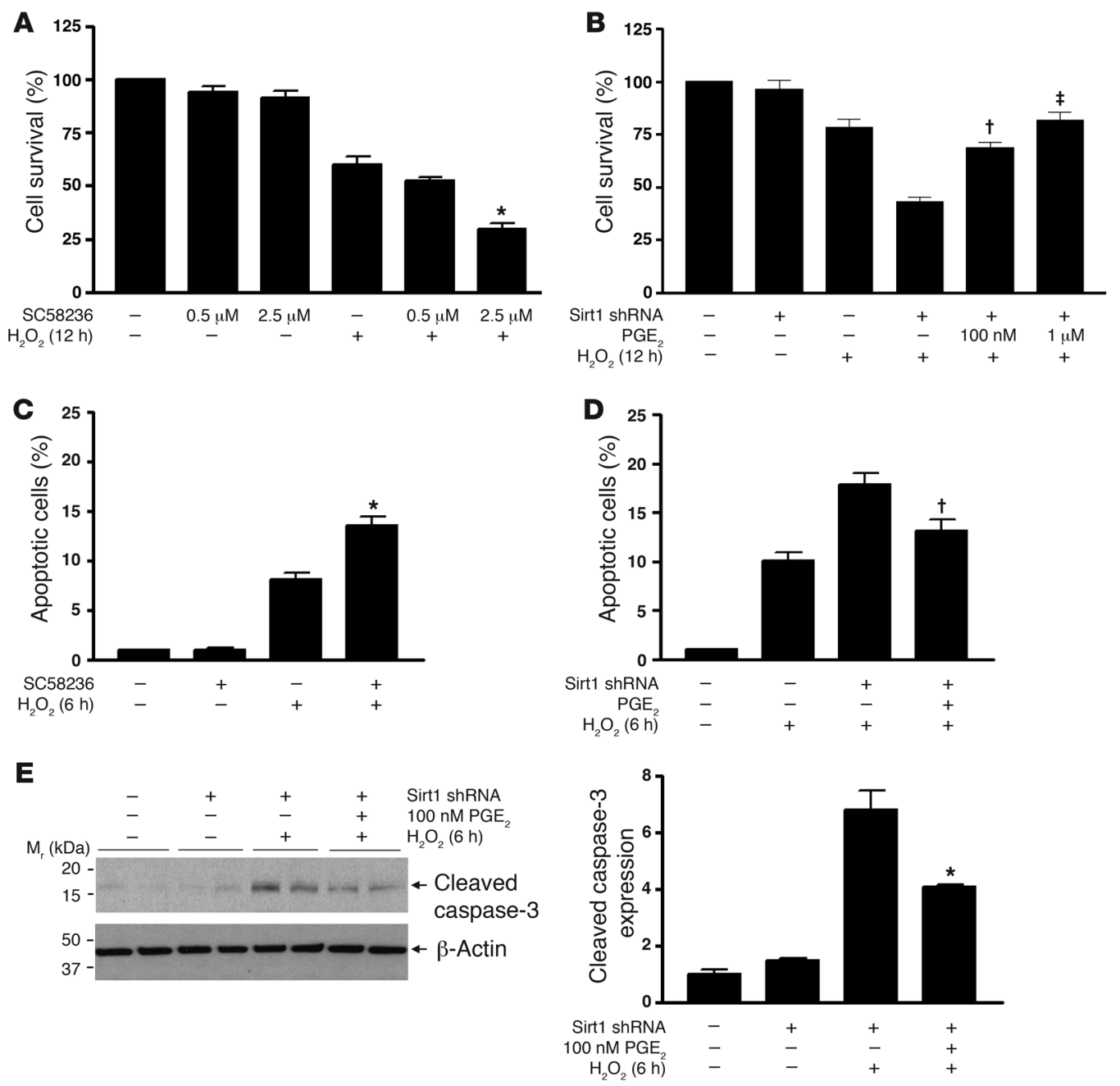

\section{Figure 9}

COX2 activity and its derived $\mathrm{PGE}_{2}$ protect cultured RMICs from oxidative stress. (A and B) Wild-type or Sirt1-knockdown cultured RMICs were pretreated with the COX2 selective inhibitor SC58236 $(0.5 \mu \mathrm{M}, 2.5 \mu \mathrm{M})$ or $\mathrm{PGE}_{2}(100 \mathrm{nM}, 1 \mu \mathrm{M})$ and challenged with $\mathrm{H}_{2} \mathrm{O}_{2}(250 \mu \mathrm{M})$ for 12 hours. Cell viability was examined by crystal violet staining $\left(n=6\right.$; ${ }^{*} P<0.0001$ versus cells with $\mathrm{H}_{2} \mathrm{O}_{2}$ alone; ${ }^{\dagger} P<0.0001$ versus Sirt1-knockdown cells with $\mathrm{H}_{2} \mathrm{O}_{2}$ without $P \mathrm{PE}_{2} ; \ddagger P<0.05$ versus Sirt1-knockdown cells with $\mathrm{H}_{2} \mathrm{O}_{2}$ and $100 \mathrm{nM} \mathrm{PGE} \mathrm{E}_{2}$ ). (C and D) Wild-type or Sirt1-knockdown RMICs were pretreated with SC58236 $(2.5 \mu \mathrm{M})$ or $\mathrm{PGE}_{2}(100 \mathrm{nM})$ and challenged with $\mathrm{H}_{2} \mathrm{O}_{2}(500 \mu \mathrm{M})$ for 6 hours. Cell apoptosis was examined by TUNEL assay $\left(n=15 ;{ }^{*} P<0.001\right.$ versus cells with $\mathrm{H}_{2} \mathrm{O}_{2}$ alone; ${ }^{\dagger} P<0.05$ versus Sirt1-knockdown cells with $\mathrm{H}_{2} \mathrm{O}_{2}$ without $\mathrm{PGE}$ ). (E) Wild-type or Sirt1knockdown RMICs were pretreated with $100 \mathrm{nM} \mathrm{PGE}_{2}$ and challenged with $\mathrm{H}_{2} \mathrm{O}_{2}(500 \mu \mathrm{M})$ for 6 hours. Expression of the cellular apoptosis marker cleaved caspase-3 was examined by immunoblot $\left(n=4\right.$, densitometry, ${ }^{*} P<0.05$ versus Sirt1-knockdown cells with $\mathrm{H}_{2} \mathrm{O}_{2}$ without $\left.P G E_{2}\right)$.

Tris-buffered saline containing $0.1 \%$ Tween-20, and incubated with a biotinylated secondary antibody for 30 minutes. After washing in PBS, sections were incubated with horseradish peroxidase-conjugated anti-biotin labeling solution (ABC Elite Kit, Vector) for 30 minutes at $22^{\circ} \mathrm{C}$, followed by washing and incubation with 3,3-diaminobenzidine solution (DAB). Counterstaining was then performed before examination under a light microscope. The primary antibodies used were as follows: anti-Sirt1 antibody (Millipore rabbit polyclonal, 1:500), anti-nitrosylated tyrosine antibody, and anti4-hydroxynonenal antibody (R\&D Biosystems mouse monoclonal, 1:500).

Immunofluorescence staining. Kidney tissues were fixed in $4 \%$ paraformaldehyde and then incubated in $30 \%$ sucrose overnight. Cryostat sections $(5 \mu \mathrm{m})$ were blocked with $3 \%$ normal donkey serum for 20 minutes and then incubated with primary antibody for 60 minutes at room temperature. After washing in PBS, the sections were incubated in Cy2- or Cy3conjugated anti-IgG secondary antibody (Jackson ImmunoResearch Labo- ratories, 1:200) for 30 minutes and washed in PBS 5 times, and microscopy was performed with a Zeiss Axioskop and spot-cam digital camera (Diagnostic Instruments) or confocal microscope (Zeiss LSM510). The primary antibodies used for immunofluorescence studies were: anti-Sirt 1 antibody (Millipore rabbit polyclonal, 1:500), anti-AQP1 antibody (Santa Cruz Biotechnology Inc. mouse monoclonal, 1:100), anti-Tamm Horsfall protein (THP) antibody (MP Biomedical goat polyclonal, 1:1,000), anti-AQP2 antibody (Santa Cruz Biotechnology Inc. goat polyclonal, 1:400), and antiCOX2 antibody (Cayman rabbit polyclonal, 1:500).

Culture of RMICs. Mouse RMICs were prepared as described previously $(9,54)$. Briefly, 8 kidneys were harvested from donor C57BL6/J mice under sterile conditions, and medullary regions were excised, minced, and suspended in DMEM (Invitrogen) containing 10\% (v/v) fetal bovine serum and penicillin/streptomycin. The pooled suspension containing renal medullary tissues from the donor mice was then injected intracutaneously at 3 or 
4 different locations in the ventral abdominal wall of an isogeneric recipient mouse. Four days later, the recipient mouse was sacrificed, and the firm yellow nodules were removed and minced under and cells trypsinized in $0.05 \%$ trypsin-EDTA at $37^{\circ} \mathrm{C}$ for 15 minutes, washed, and pelleted at $230 \mathrm{~g}$. The pellet was resuspended and cultured in DMEM supplemented with $10 \%$ fetal bovine serum and penicillin/streptomycin at $37^{\circ} \mathrm{C}$ in a $95 \%$ air $/ 5 \% \mathrm{CO}_{2}$ incubator. The cultured mouse RMICs contained oil red $\mathrm{O}$ staining-positive lipid-rich droplets, which is a characteristic feature of type 1 medullary interstitial cells (55).

Sirt1 knockdown by lentivirus carrying selective Sirt1 shRNA. HEK293T cells were cotransfected with lentiviral PLKO.1 plasmid carrying Sirt1-selective shRNA (Sigma-Aldrich MISSION shRNA library, SHCLNG-NM_019812), psPAX2 packaging plasmid, and pMD2.G envelop plasmid using FuGENE (Roche). Twelve hours later, the medium containing the transfection reagent was removed and replaced with fresh complete DMEM plus $10 \%$ FBS and penicillin/streptomycin. Twenty-four hours later, the culture medium containing lentiviral particles was harvested from HEK293T cells and transferred to a polypropylene storage tube. Virus was stored in aliquots at $-80^{\circ} \mathrm{C}$. Primary cultured mouse RMICs were then infected with appropriate amounts of lentiviral particles containing medium. Twentyfour hours later, virus-containing medium was removed and replaced with fresh medium. Infected mouse RMICs were cultured for 3 days, and then RT-PCR or immunoblot was performed to examine the efficiency of mRNA or protein knockdown. Controls included empty pLKO.1 plasmid or pLKO.1 plasmid containing scrambled shRNA. In addition, 2 different Sirt1-selective shRNAs from Sigma-Aldrich were used side by side in all the knockdown experiments, and they resulted in similar phenotypes.

$R T-P C R$. Total RNA was extracted from cultured mouse RMICs by using TRIzol reagent (Invitrogen) and reverse transcribed using high-capacity cDNA reverse transcription kit (Applied Biosystems). The primers used for PCR were Sirt1: sense 5'-GCAACAGCATCTTGCCTGAT-3', antisense 5'-GTGCTACTGGTCTCACTT-3'; SIRT2: sense 5'-CTTCCTGGGCATGATGAT-3', antisense 5'-ACCCTGACTGGGCATCTAT-3'; SIRT3: sense 5'-CAGCAACCTTCAGCAGTA-3', antisense 5'-CCGTGCATGTAGCTGTTA- $3^{\prime}$. The PCR program used 30 cycles $\left(94^{\circ} \mathrm{C}, 30\right.$ seconds; $58^{\circ} \mathrm{C}, 30$ seconds; $72^{\circ} \mathrm{C}, 45$ seconds).

qRT-PCR. Total RNA was extracted from renal tissues or cultured RMICs using TRIzol reagent (Invitrogen). Reverse transcription was performed using a high-capacity cDNA reverse transcription kit (Applied Biosystems). qRT-PCR was performed using the Taqman gene expression assay system (Applied biosystems). The probes used were Mm01168521_m1 (mouse Sirt1), Mm00478374_m1 (mouse COX2), Mm00477214_m1 (mouse COX1), Mm00801666_g1 (mouse Col1a1), Mm00802372_m1 (mouse Col4a1). Probes for eukaryotic 18S rRNA (4319413E) were used as endogenous control. Gene expression values were calculated based on the comparative threshold cycle $(\mathrm{Ct})$ method detailed in ref. 56, normalized to the expression values of $18 \mathrm{~S}$ rRNA, and displayed as fold induction relative to control.

Crystal violet staining. Cell viability was assessed using crystal violet staining (57-59). Culture medium was first removed, and culture plates were washed with PBS. The remaining viable attached cells were stained with $0.5 \%$ crystal violet in $50 \%$ methanol for 15 minutes at room temperature and gently rinsed with water and dried. Crystal violet in each well of 12-well plate was then re-dissolved by $500 \mu \mathrm{l}$ of $0.1 \mathrm{M}$ citrate sodium in $20 \%$ methanol, pH 5.4. Thirty minutes later, the absorbance at $570 \mathrm{~nm}$ was read using a spectrophotometer. For controls, cells were left untreated. Survival in the control wells with no treatment was then arbitrarily set as $100 \%$, and the crystal violet absorbance in treated wells was compared with that in control wells to obtain the relative cell survival percentage.

TUNEL. TUNEL assay for cultured RMICs was performed according to the manufacturer's protocols (In Situ Cell Death Detection Kit, Fluores- cein, catalog 11684795910, Roche). Vectashield mounting medium for fluorescence with DAPI (Vector) was used. Immunofluorescence microscopy was performed with a Zeiss Axioskop and spot-cam digital camera (Diagnostic Instruments). Apoptotic cell percentage was quantified by calculating the ratio of TUNEL-positive cells to DAPI-stained total nuclei on at least 15 random high-power fields (HPFs, $\times 400$ at final magnification).

For TUNEL assay on kidney sections, 5 - $\mu \mathrm{m}$-thick sections of paraffinembedded tissue were dewaxed and hydrated, quenched in $3 \% \mathrm{H}_{2} \mathrm{O}_{2}$ for 15 minutes to remove endogenous hydroxyl peroxidase activity, and then subjected to microwave antigen retrieval and proteinase $\mathrm{K}$ treatment to expose DNA. Slides were incubated in a humidified chamber for 1 hour at room temperature in a reaction solution containing $\mathrm{TdT}$ terminal transferase (Fisher), Bio-14-dATP (Gibco, Invitrogen), and One-Phor-All buffer (supplied with TdT terminal transferase; Fisher). The reaction was terminated by two 3 -minute washes in PBS, followed by $2 \%$ BSA in water for 10 minutes at room temperature, and incubated in horseradish peroxidase-conjugated anti-biotin labeling solution (ABC Elite kit, Vector) and stained with DAB. Quantification of apoptotic cells was performed by counting TUNEL-positive cells in at least 6 random HPFs in the renal medulla (including outer and inner medulla) of one kidney section.

Sirius red staining. Collagen accumulation in kidney sections was determined by staining for Sirius red (60) and quantified by image analysis. Analysis was made without knowledge of the treatment protocol. In brief, paraffin-embedded sections were cut into $5-\mu \mathrm{m}$-thick sections and deparaffinized. The slides were incubated in picrosirius red solution $(0.5 \mathrm{~g}$ Sirius red F3B [C.I. 35782, Sigma-Aldrich] in $500 \mathrm{ml}$ saturated aqueous solution of picric acid) for 1 hour, and then washed in 2 changes of acidified water $(5 \mathrm{ml}$ acetic acid in 11 water). After physical removal of most of the water from the slides by vigorous shaking or blotting with dam filter paper, the slides were dehydrated in 3 changes of $100 \%$ ethanol, cleared in xylene, and mounted in a resinous medium. A Zeiss Axioskop and spot-cam digital camera (Diagnostic Instruments) were used to capture 15-25 non-overlapping fields per one kidney section at a final magnification of $\times 200$. Image analysis was performed using ImageJ with modifications of techniques described previously (61). Data are presented as the mean tubulointerstitial area occupied by collagen fibrils reactive with Sirius red compared with total area (\%).

Dual luciferase reporter assay. The 891-bp human COX2 promoter-driven Firefly luciferase reporter construct was provided by Lee-Ho Wang (Department of Hematology, University of Texas Health Science Center at Houston) (62). The COX2 reporter Firefly luciferase plasmid and a plasmid containing Renilla luciferase driven by the TK promoter (Promega) were cotransfected into primary cultured mouse RMICs using FuGENE (Roche). Dual Luciferase assay kit (Promega) was used to measure both Firefly and Renilla luciferase activity in the transfected RMICs with or without treatment. Relative luciferase activity was defined as COX2 reporter Firefly luciferase activity adjusted by Renilla luciferase activity. Data are presented as fold induction compared with control group.

ChIP assay. ChIP assay was performed according to the manufacturer's protocol (Millipore, ChIP Assay kit, catalog 17-295). Cultured mouse RMICs were cross-linked by $1 \%$ formaldehyde and incubated for $10 \mathrm{~min}$ utes at $37^{\circ} \mathrm{C}$. After washing twice using ice cold PBS containing protease inhibitors, cells were scraped into conical tubes and spun down for $4 \mathrm{~min}$ utes at $640 \mathrm{~g}$ at $4{ }^{\circ} \mathrm{C}$. The cell pellet was resuspended in SDS lysis buffer and incubated for 10 minutes on ice. The lysates were sonicated on ice to shear DNA to lengths between 200 and 1,000 base pairs and then diluted 10 -fold in ChIP dilution buffer with protease inhibitors. The immunoprecipitating antibody (Millipore rabbit anti-Sirt1, working concentration $5 \mu \mathrm{g} / \mathrm{ml}$ ) was added to the supernatant fraction for overnight incubation at $4^{\circ} \mathrm{C}$ with rotation (for a negative control, preimmune IgG was added for immunoprecipitation). Then salmon sperm DNA/protein A agarose 
slurry (50 $\mu \mathrm{l}$ into $2 \mathrm{ml}$ supernatant) was added for 1-hour incubation at $4^{\circ} \mathrm{C}$ with rotation. The agarose was pelleted by gentle centrifugation $(100 \mathrm{~g}$ at $4^{\circ} \mathrm{C}, \sim 1$ minute). The supernatant containing unbound, nonspecific DNA was removed. The protein A agarose complex was washed for 3-5 minutes on a rotating platform with the washing buffers supplied with the kit. The precipitated complex was eluted from the antibody by adding freshly prepared elution buffer ( $\left.1 \% \mathrm{SDS}, 0.1 \mathrm{M} \mathrm{NaHCO}_{3}\right)$, vortexed briefly, and incubated at room temperature for 15 minutes. The agarose was then spun down, and the supernatant fraction (eluate) was transferred to another tube and elution repeated. Eluates were combined and proteinDNA crosslinks were reversed by adding $5 \mathrm{M} \mathrm{NaCl}(20 \mu \mathrm{l}$ into $500 \mu \mathrm{l})$ and heating at $65^{\circ} \mathrm{C}$ for 4 hours. The reverse-crosslinked eluates were further treated with proteinase $\mathrm{K}$, and DNA was recovered by phenol/chloroform extraction and ethanol precipitation. Pellets were washed with $70 \%$ ethanol and air dried. Pellets were then resuspended in an appropriate buffer for PCR. The primers used were as follows: $[-712,-396]$ region, sense 5 '-CAGCAGGGGGAAAATACCTT-3', antisense 5'-CGGGATCTAAGGTCCTAACT-3'; $[-4,166,-3,946]$ region, sense 5'-GGACTGGCTAGAGACATTGA-3', antisense $5^{\prime}$-AGCAGGGAACACATGGATGA- $3^{\prime}$. The PCR program used 30 cycles $\left(94^{\circ} \mathrm{C}, 30\right.$ seconds; $60^{\circ} \mathrm{C}, 1$ minute; $72^{\circ} \mathrm{C}, 1$ minute).
Statistics. Data are shown as mean \pm SEM. Statistical analysis was performed by using GraphPad Prism (GraphPad Software). An unpaired 2-tailed Student's $t$ test was used to determine the significant differences. A $P$ value less than 0.05 was considered significant.

\section{Acknowledgments}

This work is supported by NIDDK grants DK071876 and DK074116 (to C.-M. Hao) and DK44757 and DK56942 (A.B. Fogo). R. Zent is recipient of NIH grants DK075594 and DK65123, an American Heart Association established investigator award, and a merit award from the Department of Veterans Affairs. L. You is supported by an International Society of Nephrology Fellowship grant.

Received for publication October 26, 2009, and accepted in revised form February 10, 2010.

Address correspondence to: Chuan-Ming Hao, S3223 MCN, Vanderbilt University Medical Center, Nashville, Tennessee 37232. Phone: 615.873.7513; Fax: 615.343.4704; E-mail: chuanming. hao@vanderbilt.edu.
1. Hao CM, Yull F, Blackwell T, Komhoff M, Davis LS, Breyer MD. Dehydration activates an NF-kappaB-driven, COX2-dependent survival mechanism in renal medullary interstitial cells. J Clin Invest. 2000;106(8):973-982.

2. Pallone TL. Is oxidative stress differentially regulated in the renal cortex and medulla? Nat Clin Pract Nephrol. 2006;2(3):118-119.

3. Burg MB, Ferraris JD, Dmitrieva NI. Cellular response to hyperosmotic stresses. Physiol Rev. 2007;87(4):1441-1474.

4. Zhou XJ, Rakheja D, Yu X, Saxena R, Vaziri ND, Silva FG. The aging kidney. Kidney Int. 2008; 74(6):710-720.

5. Roberts CK, Sindhu KK. Oxidative stress and metabolic syndrome. Life Sci. 2009;84(21-22):705-712.

6. Haugan K, Shalmi M, Petersen JS, Marcussen N, Spannow J, Christensen S. Effects of renal papillary-medullary lesion on the antihypertensive effect of furosemide and development of salt-sensitive hypertension in Dahl-S rats. J Pharmacol Exp Ther. 1997;280(3):1415-1422.

7. Bach PH, Nguyen TK. Renal papillary necrosis -40 years on. Toxicol Pathol. 1998;26(1):73-91.

8. Harris RC. Cyclooxygenase-2 and the kidney: functional and pathophysiological implications. J Hypertens Suppl. 2002;20(6):S3-S9.

9. Hao CM, Breyer MD. Hypertension and cyclooxygenase- 2 inhibitors: target: the renal medulla. Hypertension. 2004;44(4):396-397.

10. Cowley AW Jr. Renal medullary oxidative stress, pressure-natriuresis, and hypertension. Hypertension. 2008;52(5):777-786.

11. Afshar G, Murnane JP. Characterization of a human gene with sequence homology to Saccharomyces cerevisiae SIR2. Gene. 1999;234(1):161-168.

12. Shi T, Wang F, Stieren E, Tong Q. SIRT3, a mitochondrial sirtuin deacetylase, regulates mitochondrial function and thermogenesis in brown adipocytes. J Biol Chem. 2005;280(14):13560-13567.

13. Guarente L, Picard F. Calorie restriction - the SIR2 connection. Cell. 2005;120(4):473-482.

14. Baur JA, et al. Resveratrol improves health and survival of mice on a high-calorie diet. Nature. 2006; 444(7117):337-342.

15. Milne JC, et al. Small molecule activators of SIRT1 as therapeutics for the treatment of type 2 diabetes. Nature. 2007;450(7170):712-716.

16. Kim D, et al. SIRT1 deacetylase protects against neurodegeneration in models for Alzheimer's disease and amyotrophic lateral sclerosis. EMBO J. 2007;
26(13):3169-3179.

17. Lavu S, Boss O, Elliott PJ, Lambert PD. Sirtuins - novel therapeutic targets to treat age-associated diseases. Nat Rev Drug Discov. 2008;7(10):841-853.

18. Lee JH, et al. Overexpression of SIRT1 protects pancreatic beta-cells against cytokine toxicity by suppressing the nuclear factor-kappaB signaling pathway. Diabetes. 2009;58(2):344-351.

19. Alcendor RR, et al. Sirt1 regulates aging and resistance to oxidative stress in the heart. Circ Res. 2007; 100(10):1512-1521.

20. Della-Morte D, Dave KR, Defazio RA, Bao YC, Raval AP, Perez-Pinzon MA. Resveratrol pretreatment protects rat brain from cerebral ischemic damage via a sirtuin 1 - uncoupling protein 2 pathway. NeuroScience. 2009;159(3):993-1002.

21. Chevalier RL. Obstructive nephropathy: towards biomarker discovery and gene therapy. Nat Clin Pract Nephrol. 2006;2(3):157-168.

22. Chevalier RL, Forbes MS, Thornhill BA. Ureteral obstruction as a model of renal interstitial fibrosis and obstructive nephropathy. Kidney Int. 2009;75(11):1145-1152.

23. Cheng HL, et al. Developmental defects and p53 hyperacetylation in Sir2 homolog (SIRT1)deficient mice. Proc Natl Acad Sci U S A. 2003; 100(19):10794-10799.

24. Yang T, Schnermann JB, Briggs JP. Regulation of cyclooxygenase- 2 expression in renal medulla by tonicity in vivo and in vitro. Am J Physiol. 1999; 277(1 Pt 2):F1-F9.

25. Hao CM, Komhoff M, Guan Y, Redha R, Breyer MD. Selective targeting of cyclooxygenase- 2 reveals its role in renal medullary interstitial cell survival. Am J Physiol. 1999;277(3 Pt 2):F352-F359.

26. Moeckel GW, Zhang L, Fogo AB, Hao CM, Pozzi A, Breyer MD. COX2 activity promotes organic osmolyte accumulation and adaptation of renal medullary interstitial cells to hypertonic stress. J Biol Chem. 2003;278(21):19352-19357.

27. Neuhofer W, Beck FX. Survival in hostile environments: strategies of renal medullary cells. Physiology (Bethesda). 2006;21(1-4):171-180.

28. Stern L, Backman KA, Hayslett JP. Effect of corticalmedullary gradient for ammonia on urinary excretion of ammonia. Kidney Int. 1985;27(4):652-661.

29. Zhang D, Li S, Cruz P, Kone BC. Sirtuin 1 functionally and physically interacts with disruptor of telomeric silencing- 1 to regulate alpha-ENaC transcription in collecting duct. J Biol Chem. 2009;284(31):20917-20926.
30. Hackbarth $\mathrm{H}$, Harrison DE. Changes with age in renal function and morphology in C57BL/6, CBA/HT6, and B6CBAF1 mice. J Gerontol. 1982; 37(5):540-547.

31. Davies I, Fotheringham AP, Faragher BE. Ageassociated changes in the kidney of the laboratory mouse. Age Ageing. 1989;18(2):127-133.

32. Wood JG, et al. Sirtuin activators mimic caloric restriction and delay ageing in metazoans. Nature. 2004;430(7000):686-689.

33. Bordone L, Guarente L. Calorie restriction, SIRT1 and metabolism: understanding longevity. Nat Rev Mol Cell Biol. 2005;6(4):298-305.

34. Brooks CL, Gu W. How does SIRT1 affect metabolism, senescence and cancer? Nat Rev Cancer. 2009; 9(2):123-128.

35. Ruwart MJ, Rush BD, Friedle NM, Piper RC, Kolaja GJ. Protective effects of 16,16-dimethyl PGE2 on the liver and kidney. Prostaglandins. 1981; 21(suppl):97-102.

36. Rincon-Sanchez AR, et al. PGE2 alleviates kidney and liver damage, decreases plasma renin activity and acute phase response in cirrhotic rats with acute liver damage. Exp Toxicol Pathol. 2005;56(4-5):291-303.

37 . Hodges RJ, et al. Severity of lung injury in cyclooxygenase-2-deficient mice is dependent on reduced prostaglandin E(2) production. Am J Pathol. 2004; 165(5):1663-1676.

38. Osborne NN, et al. Expression of prostaglandin PGE2 receptors under conditions of aging and stress and the protective effect of the EP2 agonist butaprost on retinal ischemia. Invest Ophthalmol Vis Sci. 2009;50(7):3238-3248.

39. Echeverria V, Clerman A, Dore S. Stimulation of PGE receptors EP2 and EP4 protects cultured neurons against oxidative stress and cell death following beta-amyloid exposure. Eur J Neurosci. 2005; 22(9):2199-2206.

40. Hao CM, Redha R, Morrow J, Breyer MD. Peroxisome proliferator-activated receptor delta activation promotes cell survival following hypertonic stress. J Biol Chem. 2002;277(24):21341-21345.

41. Hoshino T, Tsutsumi S, Tomisato W, Hwang HJ, Tsuchiya T, Mizushima T. Prostaglandin E2 protects gastric mucosal cells from apoptosis via EP2 and EP4 receptor activation. J Biol Chem. 2003; 278(15):12752-12758.

42. Liou JY, et al. Cyclooxygenase-2-derived prostaglandin e2 protects mouse embryonic stem cells from apoptosis. Stem Cells. 2007;25(5):1096-1103.

43. George RJ, Sturmoski MA, Anant S, Houchen 
CW. EP4 mediates PGE2 dependent cell survival through the PI3 kinase/AKT pathway. Prostaglandins Other Lipid Mediat. 2007;83(1-2):112-120.

44. Yeung F, et al. Modulation of NF-kappaB-dependent transcription and cell survival by the SIRT1 deacetylase. EMBOJ. 2004;23(12):2369-2380

45. Luo J, et al. Negative control of p53 by Sir2alpha promotes cell survival under stress. Cell. 2001; 107(2):137-148.

46. Brunet A, et al. Stress-dependent regulation of FOXO transcription factors by the SIRT1 deacetylase. Science. 2004;303(5666):2011-2015.

47. Rodgers JT, Lerin C, Haas W, Gygi SP, Spiegelman BM, Puigserver P. Nutrient control of glucose homeostasis through a complex of PGC-1alpha and SIRT1. Nature. 2005;434(7029):113-118.

48. Kang YJ, Mbonye UR, DeLong CJ, Wada M, Smith WL. Regulation of intracellular cyclooxygenase levels by gene transcription and protein degradation. Prog Lipid Res. 2007;46(2):108-125.

49. Yang Y, et al. SIRT1 sumoylation regulates its deacetylase activity and cellular response to genotoxic stress. Nat Cell Biol. 2007;9(11):1253-1262.

50. Sasaki T, et al. Phosphorylation regulates SIRT1 function. PLoS One. 2008;3(12):e4020.
51. Aubin MC, Lajoie C, Clement R, Gosselin H, Calderone A, Perrault LP. Female rats fed a high-fat diet were associated with vascular dysfunction and cardiac fibrosis in the absence of overt obesity and hyperlipidemia: therapeutic potential of resveratrol. J Pharmacol Exp Ther. 2008;325(3):961-968.

52. Hashem MA, Jun KY, Lee E, Lim S, Choo HY, Kwon Y. A rapid and sensitive screening system for human type I collagen with the aim of discovering potent anti-aging or anti-fibrotic compounds. $\mathrm{Mol}$ Cells. 2008;26(6):625-630.

53. Inanaga $\mathrm{K}$, et al. Resveratrol attenuates angiotensin II-induced interleukin- 6 expression and perivascular fibrosis. Hypertens Res. 2009;32(6):466-471.

54. Dunn MJ, Staley RS, Harrison M. Characterization of prostaglandin production in tissue culture of rat renal medullary cells. Prostaglandins. 1976;12(1):37-49.

55. Brenner, BM. The Kidney, 8th ed. Philadelphia, PA: W.B. Saunders Company; 2008.

56. Applied Biosystems. User Bulletin \#2: ABI PRISM 7700 Sequence Detection System. December 11, 1997. Updated October 2001.

57. Wang CY, Mayo MW, Baldwin AS Jr. TNF- and cancer therapy-induced apoptosis: potentiation by inhibition of NF-kappaB. Science. 1996;274(5288):784-787.
58. Mehlen P, Kretz-Remy C, Preville X, Arrigo AP. Human hsp27, Drosophila hsp27 and human alphaB-crystallin expression-mediated increase in glutathione is essential for the protective activity of these proteins against TNFalpha-induced cell death. EMBO J. 1996;15(11):2695-2706.

59. Rao R, Hao CM, Breyer MD. Hypertonic stress activates glycogen synthase kinase 3 beta-mediated apoptosis of renal medullary interstitial cells, suppressing an NFkappaB-driven cyclooxygenase2-dependent survival pathway. J Biol Chem. 2004; 279(6):3949-3955.

60. Lopez-De Leon A, Rojkind M. A simple micromethod for collagen and total protein determination in formalin-fixed paraffin-embedded sections.J Histochem Cytochem. 1985;33(8):737-743.

61. McKim SE, Uesugi T, Raleigh JA, McClain CJ, Arteel GE. Chronic intragastric alcohol exposure causes hypoxia and oxidative stress in the rat pancreas. Arch Biochem Biophys. 2003;417(1):34-43.

62. Tazawa R, Xu XM, Wu KK, Wang LH. Characterization of the genomic structure, chromosomal location and promoter of human prostaglandin $\mathrm{H}$ synthase-2 gene. Biochem Biophys Res Commun. 1994; 203(1):190-199. 